\title{
F-Expansion Method and New Exact Solutions of the Schrödinger-KdV Equation
}

\author{
Ali Filiz, ${ }^{1}$ Mehmet Ekici, ${ }^{2}$ and Abdullah Sonmezoglu ${ }^{2}$ \\ ${ }^{1}$ Department of Mathematics, Faculty of Science and Arts, Adnan Menderes University, 09010 Aydin, Turkey \\ ${ }^{2}$ Department of Mathematics, Faculty of Science and Arts, Bozok University, 66100 Yozgat, Turkey
}

Correspondence should be addressed to Ali Filiz; afiliz@adu.edu.tr

Received 17 August 2013; Accepted 27 October 2013; Published 29 January 2014

Academic Editors: A. K. Sharma and C. Yiu

Copyright (c) 2014 Ali Filiz et al. This is an open access article distributed under the Creative Commons Attribution License, which permits unrestricted use, distribution, and reproduction in any medium, provided the original work is properly cited.

F-expansion method is proposed to seek exact solutions of nonlinear evolution equations. With the aid of symbolic computation, we choose the Schrödinger-KdV equation with a source to illustrate the validity and advantages of the proposed method. A number of Jacobi-elliptic function solutions are obtained including the Weierstrass-elliptic function solutions. When the modulus $m$ of Jacobielliptic function approaches to 1 and 0 , soliton-like solutions and trigonometric-function solutions are also obtained, respectively. The proposed method is a straightforward, short, promising, and powerful method for the nonlinear evolution equations in mathematical physics.

\section{Introduction}

Nonlinear evolution equations are widely used to describe complex phenomena in many scientific and engineering fields, such as fluid dynamics, plasma physics, hydrodynamics, solid state physics, optical fibers, and acoustics. Therefore, finding solutions of such nonlinear evolution equations is important. However, determining solutions of nonlinear evolution equations is a very difficult task and only in certain cases one can obtain exact solutions. Recently, many powerful methods to obtain exact solutions of nonlinear evolution equations have been proposed, such as the inverse scattering method [1], the Bäcklund transformation method $[2,3]$, the Hirota bilinear scheme $[4,5]$, the Painlev expansion [6], the homotopy perturbation method $[7,8]$, the homogenous balance method [9], the variational method [10-12], the tanh function method [13-16], the trial function and the sine-cosine method [17], $\left(G^{\prime} / G\right)$-expansion method [18, 19], the trial equation method [20-28], the auxiliary equation method [29], the Jacobian-elliptic function method [30$33]$, the F-expansion method [34-38], and the Exp-function method [39-42].

In the present research, we shall apply the the F-expansion method to obtain 52 types of exact solution: six for the Weierstrass-elliptic function solutions and the rest for Jacobian-elliptic function solutions of the Schrdinger-KdV equation:

$$
i u_{t}=u_{x x}+u v, \quad v_{t}+6 v v_{x}+v_{x x x}=\left(|u|^{2}\right)_{x} .
$$

Among the methods mentioned above, the auxiliary equation method [29] is based on the assumption that the travelling wave solutions are in the form

$$
u(\eta)=\sum_{i=0}^{n} a_{i} z^{i}(\eta), \quad \eta=\alpha(x-\beta t),
$$

where $z(\eta)$ satisfies the following auxiliary ordinary differential equation:

$$
\left(\frac{d z}{d \eta}\right)^{2}=a z^{2}(\eta)+b z^{3}(\eta)+c z^{4}(\eta)
$$

where $a, b$, and $c$ are real parameters. Although many exact solutions were obtained in [29] via the auxiliary equation (3), all these solutions are expressed only in terms of hyperbolic and trigonometric functions. In this paper, we want to generalize the work in [29]. We propose a new auxiliary 
equation which has more general exact solutions in terms of Jacobian-elliptic and the Weierstrass-elliptic functions. Moreover, many exact solutions in terms of hyperbolic and trigonometric functions can be also obtained when the modulus of Jacobian-elliptic functions tends to one and zero, respectively.

The rest of the paper is arranged as follows. In Section 2, we briefly describe the auxiliary equation method ( $F$ expansion method) for nonlinear evolution equations. By using the method proposed in Section 2, Jacobian-elliptic and the Weierstrass-elliptic functions solutions are presented in Sections 3 and 4, respectively. Soliton-like solutions and trigonometric-function solutions are listed in Sections 5 and 6 , respectively. Some conclusions are given in Section 7. The paper is ended by Appendices A-D which play an important role in obtaining the solutions.

\section{Description of the $F$-Expansion Method}

Consider a nonlinear partial differential equation (PDE) with independent variables $x, t$ and dependent variable $u$ :

$$
N\left(u, u_{t}, u_{x}, u_{x x}, \ldots\right)=0 .
$$

Assume that $u(x, t)=u(\xi)$, where the wave variable $\xi=$ $x+c t$. By this, the nonlinear PDE (4) reduces to an ordinary differential equation (ODE):

$$
N\left(u, c u^{\prime}, u^{\prime}, u^{\prime \prime}, \ldots\right)=0
$$

Then we seek its solutions in the form

$$
u(\xi)=\sum_{i=0}^{m} a_{i} F^{i}(\xi),
$$

where $a_{i}, i=0,1,2, \ldots, m$, are constants to be determined, $m$ is a positive integer which can be evaluated by balancing the highest order nonlinear term $(s)$ and the highest order partial derivative of $u$ in (4), and $F(\xi)$ satisfies the following auxiliary equation:

$$
F^{\prime}(\xi)=\sigma \sqrt{P F^{4}(\xi)+Q F^{2}(\xi)+R}
$$

where $\sigma= \pm 1$ and $P, Q$, and $R$ are constants. The last equation hence holds for $F(\xi)$ :

$$
\begin{gathered}
F^{\prime \prime}=2 P F^{3}+Q F, \\
F^{\prime \prime \prime}=\left(6 P F^{2}+Q\right) F^{\prime} \\
F^{(4)}=24 P^{2} F^{5}+20 P Q F^{3}+\left(12 P R+Q^{2}\right) F \\
F^{(5)}=\left(120 P^{2} F^{4}+60 P Q F^{2}+12 P R+Q^{2}\right) F^{\prime}
\end{gathered}
$$

In Appendices $\mathrm{A}$ and $\mathrm{B}$, we present 52 types of exact solution for (7) (see [34-37, 43] for details). In fact, these exact solutions can be used to construct more exact solutions for (1).

\section{New Exact Jacobian-Elliptic Function Solutions of the Schrödinger-KdV Equation}

The coupled Schrödinger-KdV equation

$$
i u_{t}-u_{x x}-u v=0, \quad v_{t}+6 v v_{x}+v_{x x x}-\left(|u|^{2}\right)_{x}=0
$$

is known to describe various processes in dusty plasma, such as Langmuir, dust-acoustic wave, and electromagnetic waves [44-47]. Exact solution of (9) was studied by many authors [48-51]. Here the F-expansion method is applied to system (9) and gives some new solutions. Let

$$
\begin{array}{ll}
u=e^{i \theta} U(\xi), & v=V(\xi), \\
\theta=\alpha x+\beta t, & \xi=x+c t
\end{array}
$$

where $\alpha, \beta$, and $c$ are constants.

Substituting (10) into (9), we find that $c=2 \alpha$ and $V, U$ satisfy the following coupled nonlinear ordinary differential system:

$$
\begin{aligned}
& U^{\prime \prime}+\left(\beta-\alpha^{2}\right) U+U V=0, \\
& 2 \alpha V^{\prime}+6 V V^{\prime}+V^{\prime \prime \prime}-\left(U^{2}\right)^{\prime}=0 .
\end{aligned}
$$

Balancing the highest nonlinear terms and the highest order derivative terms in (11), we find $m=2$ and $n=2$. Therefore, we suppose that the solution of (11) can be expressed by

$$
\begin{aligned}
& U(\xi)=a_{0}+a_{1} F(\xi)+a_{2} F^{2}(\xi), \\
& V(\xi)=b_{0}+b_{1} F(\xi)+b_{2} F^{2}(\xi),
\end{aligned}
$$

where $a_{0}, a_{1}, a_{2}, b_{0}, b_{1}$, and $b_{2}$ are constants to be determined later and $F(\xi)$ is a solution of ODE (7). Inserting (12) into (11) with the aid of (7), the left-hand side of (11) becomes polynomials in $F(\xi)$ if canceling $F^{\prime}$ and setting the coefficients of the polynomial to zero yields a set of algebraic equations, $a_{0}, a_{1}, a_{2}, b_{0}, b_{1}$, and $b_{2}$. Solving the system of algebraic equations with the aid of Mathematica, we obtain

$$
\begin{gathered}
a_{0}=0, \quad a_{1}= \pm 2 \sqrt{P\left(Q-\alpha-3 \alpha^{2}+3 \beta\right)}, \quad a_{2}=0, \\
b_{0}=\alpha^{2}-\beta-Q, \quad b_{1}=0, \quad b_{2}=-2 P .
\end{gathered}
$$

Substituting these results into (12), we have the following formal solution of (11):

$$
\begin{gathered}
U= \pm 2 \sqrt{P\left(Q-\alpha-3 \alpha^{2}+3 \beta\right)} F(\xi), \\
V=\alpha^{2}-\beta-Q-2 P F^{2}(\xi), \quad \text { where } \xi=x+c t .
\end{gathered}
$$

With the aid of Appendix A and from the formal solution of (14) along with (10), one can deduce more general combined Jacobian-elliptic function solutions of (1). Hence, the following exact solutions are obtained. 
Case 1. $P=m^{2}, Q=-\left(1+m^{2}\right), R=1, F(\xi)=s n \xi$,

$$
\begin{gathered}
u_{1}=e^{i \theta}\left\{ \pm 2 m \sqrt{-1-m^{2}-\alpha-3 \alpha^{2}+3 \beta} \sin \xi\right\}, \\
v_{1}=\alpha^{2}-\beta+1+m^{2}-2 m^{2} \operatorname{sn}^{2} \xi .
\end{gathered}
$$

Case 2. $P=m^{2}, Q=-\left(1+m^{2}\right), R=1, F(\xi)=c d \xi$,

$$
\begin{gathered}
u_{2}=e^{i \theta}\left\{ \pm 2 m \sqrt{-1-m^{2}-\alpha-3 \alpha^{2}+3 \beta} c d \xi\right\}, \\
v_{2}=\alpha^{2}-\beta+1+m^{2}-2 m^{2} c d^{2} \xi .
\end{gathered}
$$

Case 3. $P=-m^{2}, Q=2 m^{2}-1, R=1-m^{2}, F(\xi)=c n \xi$,

$$
\begin{gathered}
u_{3}=e^{i \theta}\left\{ \pm 2 m \sqrt{-2 m^{2}+1+\alpha+3 \alpha^{2}-3 \beta} c n \xi\right\}, \\
v_{3}=\alpha^{2}-\beta-2 m^{2}+1+2 m^{2} c n^{2} \xi .
\end{gathered}
$$

Case 4. $P=-1, Q=2-m^{2}, R=m^{2}-1, F(\xi)=d n \xi$,

$$
\begin{gathered}
u_{4}=e^{i \theta}\left\{ \pm 2 \sqrt{-2+m^{2}+\alpha+3 \alpha^{2}-3 \beta} d n \xi\right\}, \\
v_{4}=\alpha^{2}-\beta-2+m^{2}+2 d n^{2} \xi .
\end{gathered}
$$

Case 5. $P=1, Q=-\left(1+m^{2}\right), R=m^{2}, F(\xi)=n s \xi$,

$$
\begin{gathered}
u_{5}=e^{i \theta}\left\{ \pm 2 \sqrt{-1-m^{2}-\alpha-3 \alpha^{2}+3 \beta} n s \xi\right\}, \\
v_{5}=\alpha^{2}-\beta+1+m^{2}-2 n s^{2} \xi .
\end{gathered}
$$

Case 6. $P=1, Q=-\left(1+m^{2}\right), R=m^{2}, F(\xi)=d c \xi$,

$$
\begin{gathered}
u_{6}=e^{i \theta}\left\{ \pm 2 \sqrt{-1-m^{2}-\alpha-3 \alpha^{2}+3 \beta} d c \xi\right\}, \\
v_{6}=\alpha^{2}-\beta+1+m^{2}-2 d c^{2} \xi .
\end{gathered}
$$

Case 7. $P=1-m^{2}, Q=2 m^{2}-1, R=-m^{2}, F(\xi)=n c \xi$,

$$
\begin{gathered}
u_{7}=e^{i \theta}\left\{ \pm 2 \sqrt{\left(1-m^{2}\right)\left(2 m^{2}-1-\alpha-3 \alpha^{2}+3 \beta\right)} n c \xi\right\}, \\
v_{7}=\alpha^{2}-\beta-2 m^{2}+1-2\left(1-m^{2}\right) n c^{2} \xi .
\end{gathered}
$$

Case 8. $P=m^{2}-1, Q=2-m^{2}, R=-1, F(\xi)=n d \xi$,

$$
\begin{gathered}
u_{8}=e^{i \theta}\left\{ \pm 2 \sqrt{\left(m^{2}-1\right)\left(2-m^{2}-\alpha-3 \alpha^{2}+3 \beta\right)} n d \xi\right\} \\
v_{8}=\alpha^{2}-\beta-2+m^{2}-2\left(m^{2}-1\right) n d^{2} \xi .
\end{gathered}
$$

Case 9. $P=1-m^{2}, Q=2-m^{2}, R=1, F(\xi)=s c \xi$,

$$
\begin{gathered}
u_{9}=e^{i \theta}\left\{ \pm 2 \sqrt{\left(1-m^{2}\right)\left(2-m^{2}-\alpha-3 \alpha^{2}+3 \beta\right)} s c \xi\right\}, \\
v_{9}=\alpha^{2}-\beta-2+m^{2}-2\left(1-m^{2}\right) s c^{2} \xi .
\end{gathered}
$$

Case 10. $P=-m^{2}\left(1-m^{2}\right), Q=2 m^{2}-1, R=1, F(\xi)=s d \xi$,

$$
\begin{gathered}
u_{10}=e^{i \theta}\left\{ \pm 2 m \sqrt{\left(m^{2}-1\right)\left(2 m^{2}-1-\alpha-3 \alpha^{2}+3 \beta\right)} s d \xi\right\}, \\
v_{10}=\alpha^{2}-\beta-2 m^{2}+1+2 m^{2}\left(1-m^{2}\right) s d^{2} \xi .
\end{gathered}
$$

Case 11. $P=1, Q=2-m^{2}, R=1-m^{2}, F(\xi)=c s \xi$,

$$
\begin{gathered}
u_{11}=e^{i \theta}\left\{ \pm 2 \sqrt{2-m^{2}-\alpha-3 \alpha^{2}+3 \beta} c s \xi\right\}, \\
v_{11}=\alpha^{2}-\beta-2+m^{2}-2 c s^{2} \xi .
\end{gathered}
$$

Case 12. $P=1, Q=2 m^{2}-1, R=-m^{2}\left(1-m^{2}\right), F(\xi)=d s \xi$,

$$
\begin{gathered}
u_{12}=e^{i \theta}\left\{ \pm 2 \sqrt{2 m^{2}-1-\alpha-3 \alpha^{2}+3 \beta} d s \xi\right\}, \\
v_{12}=\alpha^{2}-\beta-2 m^{2}+1-2 d s^{2} \xi .
\end{gathered}
$$

Case 13. $P=1 / 4, Q=\left(1-2 m^{2}\right) / 2, R=1 / 4, F(\xi)=n s \xi \pm c s \xi$,

$$
\begin{gathered}
u_{13}=e^{i \theta}\left\{ \pm \sqrt{\frac{1-2 m^{2}-2 \alpha-6 \alpha^{2}+6 \beta}{2}}(n s \xi \pm c s \xi)\right\}, \\
v_{13}=\frac{1}{2}\left\{2 \alpha^{2}-2 \beta-1+2 m^{2}-(n s \xi \pm c s \xi)^{2}\right\} .
\end{gathered}
$$

Case 14. $P=\left(1-m^{2}\right) / 4, Q=\left(1+m^{2}\right) / 2, R=\left(1-m^{2}\right) / 4$, $F(\xi)=n c \xi \pm s c \xi$,

$$
\begin{aligned}
u_{14}=e^{i \theta}\{ \pm & \sqrt{\frac{\left(1-m^{2}\right)\left(1+m^{2}-2 \alpha-6 \alpha^{2}+6 \beta\right)}{2}} \\
& \times(n c \xi \pm s c \xi)\},
\end{aligned}
$$

$$
v_{14}=\frac{1}{2}\left\{2 \alpha^{2}-2 \beta-1-m^{2}-\left(1-m^{2}\right)(n c \xi \pm s c \xi)^{2}\right\} .
$$

Case 15. $P=1 / 4, Q=\left(m^{2}-2\right) / 2, R=m^{2} / 4, F(\xi)=n s \xi \pm d s \xi$,

$$
u_{15}=e^{i \theta}\left\{ \pm \sqrt{\frac{m^{2}-2-2 \alpha-6 \alpha^{2}+6 \beta}{2}}(n s \xi \pm d s \xi)\right\}
$$

$$
v_{15}=\frac{1}{2}\left\{2 \alpha^{2}-2 \beta-m^{2}+2-(n s \xi \pm d s \xi)^{2}\right\} \text {. }
$$

Case 16. $P=m^{2} / 4, Q=\left(m^{2}-2\right) / 2, R=m^{2} / 4, F(\xi)=s n \xi \pm$ $i c n \xi$,

$$
\begin{gathered}
u_{16}=e^{i \theta}\left\{ \pm m \sqrt{\frac{m^{2}-2-2 \alpha-6 \alpha^{2}+6 \beta}{2}}(\operatorname{sn} \xi \pm i c n \xi)\right\}, \\
v_{16}=\frac{1}{2}\left\{2 \alpha^{2}-2 \beta-m^{2}+2-m^{2}(\operatorname{sn} \xi \pm i c n \xi)^{2}\right\} .
\end{gathered}
$$


Case 17. $P=m^{2} / 4, Q=\left(m^{2}-2\right) / 2, R=m^{2} / 4, F(\xi)=$ $\sqrt{1-m^{2}} s d \xi \pm c d \xi$

$$
\begin{gathered}
u_{17}=e^{i \theta}\left\{ \pm m \sqrt{\frac{m^{2}-2-2 \alpha-6 \alpha^{2}+6 \beta}{2}}\right. \\
\left.\times\left(\sqrt{1-m^{2}} s d \xi \pm c d \xi\right)\right\}, \\
v_{17}=\frac{1}{2}\left\{2 \alpha^{2}-2 \beta-m^{2}+2-m^{2}\left(\sqrt{1-m^{2}} s d \xi \pm c d \xi\right)^{2}\right\} .
\end{gathered}
$$

Case 18. $P=1 / 4, Q=\left(1-m^{2}\right) / 2, R=1 / 4, F(\xi)=m c d \xi \pm$ $i \sqrt{1-m^{2}} n d \xi$,

$$
\begin{aligned}
u_{18}=e^{i \theta}\{ & \pm \sqrt{\frac{1-m^{2}-2 \alpha-6 \alpha^{2}+6 \beta}{2}} \\
& \left.\times\left(m c d \xi \pm i \sqrt{1-m^{2}} n d \xi\right)\right\}, \\
v_{18}=\frac{1}{2}\left\{2 \alpha^{2}-2 \beta-1+m^{2}\right. & \left.-\left(m c d \xi \pm i \sqrt{1-m^{2}} n d \xi\right)^{2}\right\} .
\end{aligned}
$$

Case 19. $P=1 / 4, Q=\left(1-2 m^{2}\right) / 2, R=1 / 4, F(\xi)=m s n \xi \pm$ $i d n \xi$

$$
\begin{gathered}
u_{19}=e^{i \theta}\left\{ \pm \sqrt{\frac{1-2 m^{2}-2 \alpha-6 \alpha^{2}+6 \beta}{2}}\right. \\
\times(m s n \xi \pm i d n \xi)\}, \\
v_{19}=\frac{1}{2}\left\{2 \alpha^{2}-2 \beta-1+2 m^{2}-(m s n \xi \pm i d n \xi)^{2}\right\} .
\end{gathered}
$$

Case 20. $P=1 / 4, Q=\left(1-m^{2}\right) / 2, R=1 / 4, F(\xi)=$ $\sqrt{1-m^{2}} s c \xi \pm i d c \xi$

$$
\begin{gathered}
u_{20}=e^{i \theta}\left\{ \pm \sqrt{\frac{1-m^{2}-2 \alpha-6 \alpha^{2}+6 \beta}{2}}\right. \\
\left.\times\left(\sqrt{1-m^{2}} s c \xi \pm i d c \xi\right)\right\}, \\
v_{20}=\frac{1}{2}\left\{2 \alpha^{2}-2 \beta-1+m^{2}-\left(\sqrt{1-m^{2}} s c \xi \pm i d c \xi\right)^{2}\right\} .
\end{gathered}
$$

Case 21. $P=\left(m^{2}-1\right) / 4, Q=\left(m^{2}+1\right) / 2, R=\left(m^{2}-1\right) / 4$, $F(\xi)=m s d \xi \pm n d \xi$

$$
\begin{aligned}
u_{21}=e^{i \theta}\left\{ \pm \sqrt{\frac{\left(m^{2}-1\right)\left(m^{2}+1-2 \alpha-6 \alpha^{2}+6 \beta\right)}{2}}\right. \\
\quad \times(m s d \xi \pm n d \xi)\}, \\
v_{21}=\frac{1}{2}\left\{2 \alpha^{2}-2 \beta-m^{2}-1-\left(m^{2}-1\right)\right. \\
\left.\quad \times(m s d \xi \pm n d \xi)^{2}\right\} .
\end{aligned}
$$

Case 22. $P=m^{2} / 4, Q=\left(m^{2}-2\right) / 2, R=1 / 4, F(\xi)=s n \xi /(1 \pm$ $d n \xi)$,

$$
\begin{aligned}
& u_{22}=e^{i \theta}\left\{ \pm m \sqrt{\frac{m^{2}-2-2 \alpha-6 \alpha^{2}+6 \beta}{2}} \frac{s n \xi}{1 \pm d n \xi}\right\} \\
& v_{22}=\frac{1}{2}\left\{2 \alpha^{2}-2 \beta-m^{2}+2-m^{2}\left(\frac{s n \xi}{1 \pm d n \xi}\right)^{2}\right\} .
\end{aligned}
$$

Case 23. $P=-1 / 4, Q=\left(m^{2}+1\right) / 2, R=\left(1-m^{2}\right)^{2} / 4, F(\xi)=$ $m c n \xi \pm d n \xi$,

$$
\begin{gathered}
u_{23}=e^{i \theta}\left\{ \pm \sqrt{\frac{-m^{2}-1+2 \alpha+6 \alpha^{2}-6 \beta}{2}}(m c n \xi \pm d n \xi)\right\}, \\
v_{23}=\frac{1}{2}\left\{2 \alpha^{2}-2 \beta-m^{2}-1+(m c n \xi \pm d n \xi)^{2}\right\} .
\end{gathered}
$$

Case 24. $P=\left(1-m^{2}\right)^{2} / 4, Q=\left(m^{2}+1\right) / 2, R=1 / 4, F(\xi)=$ $d s \xi \pm c s \xi$

$$
\begin{gathered}
u_{24}=e^{i \theta}\left\{ \pm\left(1-m^{2}\right) \sqrt{\frac{m^{2}+1-2 \alpha-6 \alpha^{2}+6 \beta}{2}}\right. \\
\quad \times(d s \xi \pm c s \xi)\}, \\
v_{24}=\frac{1}{2}\left\{2 \alpha^{2}-2 \beta-m^{2}-1-\left(1-m^{2}\right)^{2}(d s \xi \pm c s \xi)^{2}\right\} .
\end{gathered}
$$


Case 25. $P=m^{4}\left(1-m^{2}\right) / 2\left(2-m^{2}\right), Q=2\left(1-m^{2}\right) /\left(m^{2}-2\right)$, $R=\left(1-m^{2}\right) / 2\left(2-m^{2}\right), F(\xi)=d c \xi \pm \sqrt{1-m^{2}} n c \xi$,

$$
\begin{aligned}
& u_{25} \\
&=e^{i \theta}\{ \pm \frac{\sqrt{2} m^{2}}{2-m^{2}} \\
& \times \sqrt{\left(m^{2}-1\right)\left[2\left(1-m^{2}\right)+\left(m^{2}-2\right)\left(-\alpha-3 \alpha^{2}+3 \beta\right)\right]} \\
& \times\left.\left(d c \xi \pm \sqrt{1-m^{2}} n c \xi\right)\right\}, \\
& v_{25} \frac{1}{m^{2}-2}\left\{\left(m^{2}-2\right)\left(\alpha^{2}-\beta\right)+\left(1-m^{2}\right)\right. \\
&\left.\times\left[-2+m^{4}\left(d c \xi \pm \sqrt{1-m^{2}} n c \xi\right)^{2}\right]\right\} .
\end{aligned}
$$

Case 26. $P>0, Q<0, R=m^{2} Q^{2} /\left(1+m^{2}\right)^{2} P, F(\xi)=$ $\sqrt{-m^{2} \mathrm{Q} /\left(1+m^{2}\right) P} \operatorname{sn}\left(\sqrt{-\mathrm{Q} /\left(1+m^{2}\right)} \xi\right)$,

$$
\begin{aligned}
u_{26}=e^{i \theta}\{ & \pm 2 m \sqrt{\frac{Q\left(-Q+\alpha+3 \alpha^{2}-3 \beta\right)}{1+m^{2}}} \\
& \left.\times \operatorname{sn}\left(\sqrt{\frac{-Q}{1+m^{2}}} \xi\right)\right\}, \\
v_{26}=\alpha^{2}-\beta & -Q+\frac{2 m^{2} Q}{m^{2}+1} s n^{2}\left(\sqrt{\frac{-Q}{1+m^{2}}} \xi\right) .
\end{aligned}
$$

Case 27. $P<0, Q>0, R=\left(1-m^{2}\right) Q^{2} /\left(m^{2}-2\right)^{2} P, F(\xi)=$ $\sqrt{-Q /\left(2-m^{2}\right) P} d n\left(\sqrt{Q /\left(2-m^{2}\right)} \xi\right)$,

$$
\begin{aligned}
u_{27}=e^{i \theta}\{ & \pm 2 \sqrt{\frac{Q\left(-Q+\alpha+3 \alpha^{2}-3 \beta\right)}{2-m^{2}}} \\
& \times d n\left(\sqrt{\left.\left.\frac{Q}{2-m^{2}} \xi\right)\right\},}\right. \\
v_{27}=\alpha^{2}-\beta & -Q+\frac{2 Q}{2-m^{2}} d n^{2}\left(\sqrt{\frac{Q}{2-m^{2}}} \xi\right) .
\end{aligned}
$$

Case 28. $P<0, Q>0, R=m^{2}\left(m^{2}-1\right) Q^{2} /\left(2 m^{2}-1\right)^{2} P$, $F(\xi)=\sqrt{-m^{2} \mathrm{Q} /\left(2 m^{2}-1\right) P} c n\left(\sqrt{\mathrm{Q} /\left(2 m^{2}-1\right)} \xi\right)$,

$$
\begin{aligned}
u_{28}=e^{i \theta}\{ & \pm 2 m \sqrt{\frac{Q\left(-Q+\alpha+3 \alpha^{2}-3 \beta\right)}{2 m^{2}-1}} c n \\
& \times\left(\sqrt{\left.\left.\frac{Q}{2 m^{2}-1} \xi\right)\right\},}\right. \\
v_{28}=\alpha^{2}-\beta & -Q+\frac{2 m^{2} Q}{2 m^{2}-1} c n^{2}\left(\sqrt{\frac{Q}{2 m^{2}-1}} \xi\right) .
\end{aligned}
$$

Case 29. $P=1, Q=2-4 m^{2}, R=1, F(\xi)=s n \xi d n \xi / c n \xi$,

$$
\begin{gathered}
u_{29}=e^{i \theta}\left\{ \pm 2 \sqrt{2-4 m^{2}-\alpha-3 \alpha^{2}+3 \beta} \frac{\operatorname{sn} \xi d n \xi}{c n \xi}\right\}, \\
v_{29}=\alpha^{2}-\beta-2+4 m^{2}-\frac{2 s n^{2} \xi d n^{2} \xi}{c n^{2} \xi} .
\end{gathered}
$$

Case 30. $P=m^{2}, Q=2, R=1, F(\xi)=s n \xi c n \xi / d n \xi$,

$$
\begin{gathered}
u_{30}=e^{i \theta}\left\{ \pm 2 m \sqrt{2-\alpha-3 \alpha^{2}+3 \beta} \frac{\operatorname{sn} \xi c n \xi}{d n \xi}\right\}, \\
v_{30}=\alpha^{2}-\beta-2-\frac{2 m^{2} s n^{2} \xi c n^{2} \xi}{d n^{2} \xi} .
\end{gathered}
$$

Case 31. $P=1, Q=m^{2}+2, R=1-2 m^{2}+m^{4}, F(\xi)=$ $d n \xi c n \xi / s n \xi$,

$$
\begin{gathered}
u_{31}=e^{i \theta}\left\{ \pm 2 \sqrt{m^{2}+2-\alpha-3 \alpha^{2}+3 \beta} \frac{d n \xi c n \xi}{s n \xi}\right\} \\
v_{31}=\alpha^{2}-\beta-m^{2}-2-\frac{2 d n^{2} \xi c n^{2} \xi}{s n^{2} \xi}
\end{gathered}
$$

Case 32. $P=A^{2}(m-1)^{2} / 4, Q=\left(m^{2}+1\right) / 2+3 m, R=(m-$ $1)^{2} / 4 A^{2}, F(\xi)=d n \xi c n \xi / A(1+s n \xi)(1+m s n \xi)$,

$$
\begin{array}{r}
u_{32}=e^{i \theta}\left\{ \pm(m-1) \sqrt{\frac{m^{2}+6 m+1-2 \alpha}{2}}\right. \\
\left.\quad \times \frac{d n \xi c n \xi}{(1+s n \xi)(1+m s n \xi)}\right\}, \\
v_{32}=\frac{1}{2}\left\{2 \alpha^{2}-2 \beta-m^{2}-6 m-1\right. \\
\left.\quad-\frac{(m-1)^{2} d n^{2} \xi c n^{2} \xi}{(1+s n \xi)^{2}(1+m s n \xi)^{2}}\right\} .
\end{array}
$$

Case 33. $P=A^{2}(m+1)^{2} / 4, Q=\left(m^{2}+1\right) / 2-3 m, R=(m+$ $1)^{2} / 4 A^{2}, F(\xi)=d n \xi c n \xi / A(1+s n \xi)(1-m s n \xi)$,

$$
\begin{aligned}
u_{33}=e^{i \theta}\left\{ \pm(m+1) \sqrt{\frac{m^{2}-6 m+1-2 \alpha-6 \alpha^{2}+6 \beta}{2}}\right. & \\
& \left.\times \frac{d n \xi c n \xi}{(1+s n \xi)(1-m s n \xi)}\right\}, \\
v_{33}=\frac{1}{2}\left\{2 \alpha^{2}-2 \beta-m^{2}+6 m-1\right. & \left.-\frac{(m+1)^{2} d n^{2} \xi c n^{2} \xi}{(1+s n \xi)^{2}(1-m s n \xi)^{2}}\right\} .
\end{aligned}
$$


Case 34. $P=-4 / m, Q=6 m-m^{2}-1, R=-2 m^{3}+m^{4}+m^{2}$, $F(\xi)=m c n \xi d n \xi /\left(m s n^{2} \xi+1\right)$,

$$
\begin{gathered}
u_{34}=e^{i \theta}\left\{ \pm 4 \sqrt{m^{2}-6 m+1+\alpha+3 \alpha^{2}-3 \beta} \frac{\sqrt{m} c n \xi d n \xi}{m s n^{2} \xi+1}\right\}, \\
v_{34}=\alpha^{2}-\beta+m^{2}-6 m+1+\frac{8 m c n^{2} \xi d n^{2} \xi}{\left(m s n^{2} \xi+1\right)^{2}} .
\end{gathered}
$$

Case 35. $P=4 / m, Q=-6 m-m^{2}-1, R=2 m^{3}+m^{4}+m^{2}$, $F(\xi)=m c n \xi d n \xi /\left(m s n^{2} \xi-1\right)$,

$$
\begin{aligned}
& u_{35}=e^{i \theta}\{ \pm 4 \sqrt{-m^{2}-6 m-1-\alpha-3 \alpha^{2}+3 \beta} \\
&\left.\times \frac{\sqrt{m} c n \xi d n \xi}{m s n^{2} \xi-1}\right\}, \\
& v_{35}=\alpha^{2}-\beta+m^{2}+6 m+1-\frac{8 m c n^{2} \xi d n^{2} \xi}{\left(m s n^{2} \xi-1\right)^{2}} .
\end{aligned}
$$

Case 36. $P=1 / 4, Q=\left(1-2 m^{2}\right) / 2, R=1 / 4, F(\xi)=s n \xi /(1 \pm$ $\mathrm{cn} \xi)$,

$$
\begin{gathered}
u_{36}=e^{i \theta}\left\{ \pm \sqrt{\frac{1-2 m^{2}-2 \alpha-6 \alpha^{2}+6 \beta}{2}} \frac{s n \xi}{1 \pm c n \xi}\right\}, \\
v_{36}=\frac{1}{2}\left\{2 \alpha^{2}-2 \beta-1+2 m^{2}-\frac{s n^{2} \xi}{(1 \pm c n \xi)^{2}}\right\} .
\end{gathered}
$$

Case 37. $P=\left(1-m^{2}\right) / 4, Q=\left(1+m^{2}\right) / 2, R=\left(1-m^{2}\right) / 4$, $F(\xi)=c n \xi /(1 \pm s n \xi)$,

$$
\begin{aligned}
u_{37}=e^{i \theta}\{ & \pm \sqrt{\frac{\left(1-m^{2}\right)\left(1+m^{2}-2 \alpha-6 \alpha^{2}+6 \beta\right)}{2}} \\
& \left.\times \frac{c n \xi}{1 \pm \operatorname{sn} \xi}\right\}, \\
v_{37}= & \frac{1}{2}\left\{2 \alpha^{2}-2 \beta-1-m^{2}-\frac{\left(1-m^{2}\right) c n^{2} \xi}{(1 \pm s n \xi)^{2}}\right\} .
\end{aligned}
$$

Case 38. $P=4 m_{1}, Q=2+6 m_{1}-m^{2}, R=2+2 m_{1}-m^{2}$, $F(\xi)=m^{2} \operatorname{sn} \xi c n \xi /\left(m_{1}-d n^{2} \xi\right)$,

$$
\begin{aligned}
u_{38}=e^{i \theta}\{ & \pm 4 \sqrt{m_{1}\left(2+6 m_{1}-m^{2}-\alpha-3 \alpha^{2}+3 \beta\right)} \\
& \left.\times \frac{m^{2} s n \xi c n \xi}{m_{1}-d n^{2} \xi}\right\}, \\
v_{38}= & \alpha^{2}-\beta-2-6 m_{1}+m^{2}-\frac{8 m_{1} m^{4} s n^{2} \xi c n^{2} \xi}{\left(m_{1}-d n^{2} \xi\right)^{2}} .
\end{aligned}
$$

Case 39. $P=-4 m_{1}, Q=2-6 m_{1}-m^{2}, R=2-2 m_{1}-m^{2}$, $F(\xi)=-m^{2} \operatorname{sn} \xi \operatorname{cn} \xi /\left(m_{1}+d n^{2} \xi\right)$,

$$
\begin{aligned}
u_{39}=e^{i \theta}\{ & \pm 4 \sqrt{m_{1}\left(-2+6 m_{1}+m^{2}+\alpha+3 \alpha^{2}-3 \beta\right)} \\
& \left.\times \frac{m^{2} \operatorname{sn} \xi c n \xi}{m_{1}+d n^{2} \xi}\right\},
\end{aligned}
$$

$$
v_{39}=\alpha^{2}-\beta-2+6 m_{1}+m^{2}+\frac{8 m_{1} m^{4} s n^{2} \xi c n^{2} \xi}{\left(m_{1}+d n^{2} \xi\right)^{2}} \text {. }
$$

Case 40. $P=\left(2-m^{2}-2 m_{1}\right) / 4, Q=m^{2} / 2-1-3 m_{1}, R=(2-$ $\left.m^{2}-2 m_{1}\right) / 4, F(\xi)=m^{2} \operatorname{sn} \xi c n \xi /\left(s n^{2} \xi+\left(1+m_{1}\right) d n \xi-1-m_{1}\right)$,

$$
\begin{aligned}
& u_{40} e^{i \theta}\left\{ \pm \sqrt{\frac{\left(2-m^{2}-2 m_{1}\right)\left(m^{2}-2-6 m_{1}-2 \alpha-6 \alpha^{2}+6 \beta\right)}{2}}\right. \\
&\left.\times \frac{m^{2} \operatorname{sn} \xi c n \xi}{s n^{2} \xi+\left(1+m_{1}\right) d n \xi-1-m_{1}}\right\}, \\
& v_{40}=\frac{1}{2}\left\{2 \alpha^{2}-2 \beta-m^{2}+2+6 m_{1}\right. \\
&\left.-\frac{m^{4}\left(2-m^{2}-2 m_{1}\right) s n^{2} \xi c n^{2} \xi}{\left[s n^{2} \xi+\left(1+m_{1}\right) d n \xi-1-m_{1}\right]^{2}}\right\} .
\end{aligned}
$$

Case 41. $P=\left(2-m^{2}+2 m_{1}\right) / 4, Q=m^{2} / 2-1+3 m_{1}, R=(2-$ $\left.m^{2}+2 m_{1}\right) / 4, F(\xi)=m^{2} s n \xi c n \xi /\left(s n^{2} \xi+\left(-1+m_{1}\right) d n \xi-1-m_{1}\right)$,

$$
\begin{aligned}
& u_{41}\left\{ \pm \sqrt{\frac{\left(2-m^{2}+2 m_{1}\right)\left(m^{2}-2+6 m_{1}-2 \alpha-6 \alpha^{2}+6 \beta\right)}{2}}\right. \\
&\left.\times \frac{m^{2} \operatorname{sn} \xi c n \xi}{s n^{2} \xi+\left(-1+m_{1}\right) d n \xi-1-m_{1}}\right\}, \\
& v_{41}=\frac{1}{2}\left\{2 \alpha^{2}-2 \beta-m^{2}+2-6 m_{1}\right. \\
&-\left.\frac{m^{4}\left(2-m^{2}+2 m_{1}\right) s n^{2} \xi c n^{2} \xi}{\left[\operatorname{sn}^{2} \xi+\left(-1+m_{1}\right) d n \xi-1-m_{1}\right]^{2}}\right\} .
\end{aligned}
$$

Case 42. $P=\left(C^{2} m^{4}-\left(B^{2}+C^{2}\right) m^{2}+B^{2}\right) / 4, Q=\left(m^{2}+1\right) / 2, R=$ $\left(m^{2}-1\right) / 4\left(C^{2} m^{2}-B^{2}\right), F(\xi)=\left(\sqrt{\left(B^{2}-C^{2}\right) /\left(B^{2}-C^{2} m^{2}\right)}+\right.$ $s n \xi) /(B c n \xi+C d n \xi)$, 


$$
\begin{aligned}
u^{i \theta}\{ \pm & \sqrt{\frac{\left[C^{2} m^{4}-\left(B^{2}+C^{2}\right) m^{2}+B^{2}\right]\left(m^{2}+1-2 \alpha-6 \alpha^{2}+6 \beta\right)}{2}} \\
& \left.\times \frac{\sqrt{\left(B^{2}-C^{2}\right) /\left(B^{2}-C^{2} m^{2}\right)}+s n \xi}{B c n \xi+C d n \xi}\right\}, \\
v_{42}= & \left\{\begin{array}{l}
2 \alpha^{2}-2 \beta-m^{2}-1-\left[C^{2} m^{4}-\left(B^{2}+C^{2}\right) m^{2}+B^{2}\right] \\
\left.\times\left(\frac{\sqrt{\left(B^{2}-C^{2}\right) /\left(B^{2}-C^{2} m^{2}\right)}+s n \xi}{B c n \xi+C d n \xi}\right)^{2}\right\}
\end{array}\right.
\end{aligned}
$$

Case 43. $P=\left(B^{2}+C^{2} m^{2}\right) / 4, Q=1 / 2-m^{2}, R=$ $1 / 4\left(B^{2}+C^{2} m^{2}\right), F(\xi)=\left(\sqrt{\left(C^{2} m^{2}+B^{2}-C^{2}\right) /\left(B^{2}+C^{2} m^{2}\right)}+\right.$ $c n \xi) /(B s n \xi+C d n \xi)$,

$$
\begin{aligned}
& u_{43}=e^{i \theta}\left\{ \pm \sqrt{\frac{\left(B^{2}+C^{2} m^{2}\right)\left(1-2 m^{2}-2 \alpha-6 \alpha^{2}+6 \beta\right)}{2}}\right. \\
&\left.\quad \times \frac{\sqrt{\left(C^{2} m^{2}+B^{2}-C^{2}\right) /\left(B^{2}+C^{2} m^{2}\right)}+c n \xi}{B s n \xi+C d n \xi}\right\}, \\
& v_{43}=\frac{1}{2}\left\{2 \alpha^{2}-2 \beta-1+2 m^{2}-\left(B^{2}+C^{2} m^{2}\right)\right. \\
&\left.\times\left(\frac{\sqrt{\left(C^{2} m^{2}+B^{2}-C^{2}\right) /\left(B^{2}+C^{2} m^{2}\right)}+c n \xi}{B s n \xi+C d n \xi}\right)^{2}\right\} .
\end{aligned}
$$

Case 44. $P=\left(B^{2}+C^{2}\right) / 4, Q=m^{2} / 2-1, R=m^{4} / 4\left(B^{2}+C^{2}\right)$, $F(\xi)=\left(\sqrt{\left(B^{2}+C^{2}-C^{2} m^{2}\right) /\left(B^{2}+C^{2}\right)}+d n \xi\right) /(B s n \xi+C c n \xi)$,

$$
\begin{aligned}
u_{44}=e^{i \theta}\left\{ \pm \sqrt{\frac{\left(B^{2}+C^{2}\right)\left(m^{2}-2-2 \alpha-6 \alpha^{2}+6 \beta\right)}{2}}\right. & \\
& \left.\times \frac{\sqrt{\left(B^{2}+C^{2}-C^{2} m^{2}\right) /\left(B^{2}+C^{2}\right)}+d n \xi}{B s n \xi+C c n \xi}\right\}, \\
v_{44}=\frac{1}{2}\left\{2 \alpha^{2}-2 \beta-m^{2}+2-\left(B^{2}+C^{2}\right)\right. & \\
& \left.\times\left(\frac{\sqrt{\left(B^{2}+C^{2}-C^{2} m^{2}\right) /\left(B^{2}+C^{2}\right)}+d n \xi}{B s n \xi+C c n \xi}\right)^{2}\right\} .
\end{aligned}
$$

Case 45. $P=-\left(m^{2}+2 m+1\right) B^{2}, Q=2 m^{2}+2, R=(2 m-$ $\left.m^{2}-1\right) / B^{2}, F(\xi)=\left(m s n^{2} \xi-1\right) / B\left(m s n^{2} \xi+1\right)$,

$$
\begin{aligned}
u_{45}= & e^{i \theta}\left\{ \pm 2(m+1) \sqrt{-2 m^{2}-2+\alpha+3 \alpha^{2}-3 \beta}\right. \\
& \left.\quad \times \frac{m s n^{2} \xi-1}{m s n^{2} \xi+1}\right\}, \\
v_{45}= & \alpha^{2}-\beta-2 m^{2}-2+2(m+1)^{2} \\
\times & \left(\frac{m s n^{2} \xi-1}{m s n^{2} \xi+1}\right)^{2} .
\end{aligned}
$$

Case 46. $P=-\left(m^{2}-2 m+1\right) B^{2}, Q=2 m^{2}+2, R=-(2 m+$ $\left.m^{2}+1\right) / B^{2}, F(\xi)=\left(m s n^{2} \xi+1\right) / B\left(m s n^{2} \xi-1\right)$,

$$
\begin{aligned}
u_{46}= & e^{i \theta}\left\{ \pm 2(m-1) \sqrt{-2 m^{2}-2+\alpha+3 \alpha^{2}-3 \beta}\right. \\
& \left.\quad \times \frac{m s n^{2} \xi+1}{m s n^{2} \xi-1}\right\}, \\
v_{46}= & \alpha^{2}-\beta-2 m^{2}-2+2(m-1)^{2} \\
& \times\left(\frac{m s n^{2} \xi+1}{m s n^{2} \xi-1}\right)^{2} .
\end{aligned}
$$

We note that there is much duplication in the list of 46 solutions in terms of Jacobian-elliptic functions. Here are some examples; using the well-known identities relating Jacobian-elliptic functions (see 121.00, 129.01, 129.02, and 129.03 in [52], e.g.) reveals that $u_{1}, u_{3}$, and $u_{4}$ are identical; $v_{1}, v_{3}$, and $v_{4}$ are identical; $u_{2}, u_{8}$, and $u_{10}$ are identical; $v_{2}$, $v_{8}$, and $v_{10}$ are identical; $u_{5}, u_{11}$, and $u_{12}$ are identical; $v_{5}, v_{11}$, and $v_{12}$ are identical; $u_{6}, u_{7}$, and $u_{9}$ are identical; $v_{6}, v_{7}$, and $v_{9}$ are identical. Use of 162.01 in [52] reveals that $u_{27}$ and $u_{28}$ are equivalent and $v_{27}$ and $v_{28}$ are equivalent.

\section{The New Weierstrass-Elliptic Function Solutions of the Schrödinger-KdV Equation}

On using the solutions given in [43], mentioned in Appendix B, and from the formal solution (14) along with (10), we get then the following exact solutions.

Case 47. $g_{2}=(4 / 3)\left(Q^{2}-3 P R\right), g_{3}=(4 Q / 27)\left(-2 Q^{2}+9 P R\right)$, $F(\xi)=\sqrt{(1 / P)\left[\wp\left(\xi ; g_{2}, g_{3}\right)-(1 / 3) Q\right]}$,

$$
\begin{aligned}
& u_{47}=e^{i \theta}\{ \pm 2 \sqrt{P\left(Q-\alpha-3 \alpha^{2}+3 \beta\right)} \\
&\left.\times \sqrt{\frac{1}{P}\left[\wp\left(\xi ; g_{2}, g_{3}\right)-\frac{1}{3} Q\right]}\right\}, \\
& v_{47}=\alpha^{2}-\beta-Q-2\left[\wp\left(\xi ; g_{2}, g_{3}\right)-\frac{1}{3} Q\right] .
\end{aligned}
$$


Case 48. $g_{2}=(4 / 3)\left(Q^{2}-3 P R\right), g_{3}=(4 Q / 27)\left(-2 Q^{2}+9 P R\right)$, $F(\xi)=\sqrt{3 R /\left(3 \wp\left(\xi ; g_{2}, g_{3}\right)-Q\right)}$,

$$
\begin{gathered}
u_{48}=e^{i \theta}\left\{ \pm 2 \sqrt{P\left(Q-\alpha-3 \alpha^{2}+3 \beta\right)}\right. \\
\left.\times \sqrt{\left.\frac{3 R}{3 \wp\left(\xi ; g_{2}, g_{3}\right)-Q}\right\}}\right\} \\
v_{48}=\alpha^{2}-\beta-Q-\frac{6 P R}{3 \wp\left(\xi ; g_{2}, g_{3}\right)-Q} .
\end{gathered}
$$

Case 49. $g_{2}=-\left(5 Q D+4 Q^{2}+33 P Q R\right) / 12, g_{3}=\left(21 Q^{2}\right.$ $\left.D-63 P R D+20 Q^{3}-27 P Q R\right) / 216, F(\xi)=$ $\sqrt{12 R \wp\left(\xi ; g_{2}, g_{3}\right)+2 R(2 Q+D)} /\left(12 \wp\left(\xi ; g_{2}, g_{3}\right)+D\right)$,

$$
\begin{aligned}
u_{49}=e^{i \theta}\{ & \pm 2 \sqrt{P\left(Q-\alpha-3 \alpha^{2}+3 \beta\right)} \\
& \left.\times \frac{\sqrt{12 R \wp\left(\xi ; g_{2}, g_{3}\right)+2 R(2 Q+D)}}{12 \wp\left(\xi ; g_{2}, g_{3}\right)+D}\right\},
\end{aligned}
$$

$$
\begin{aligned}
v_{49}= & \alpha^{2}-\beta-Q \\
& -\frac{4 P R\left[6 \wp\left(\xi ; g_{2}, g_{3}\right)+2 Q+D\right]}{\left[12 \wp\left(\xi ; g_{2}, g_{3}\right)+D\right]^{2}} .
\end{aligned}
$$

Case 50. $g_{2}=(1 / 12) Q^{2}+P R, g_{3}=(1 / 216) Q\left(36 P R-Q^{2}\right)$, $F(\xi)=\sqrt{R}\left[6 \wp\left(\xi ; g_{2}, g_{3}\right)+Q\right] / 3 \wp^{\prime}\left(\xi ; g_{2}, g_{3}\right)$,

$$
\begin{aligned}
& u_{50}=e^{i \theta}\{ \pm 2 \sqrt{P R\left(Q-\alpha-3 \alpha^{2}+3 \beta\right)} \\
&\left.\times \frac{6 \wp\left(\xi ; g_{2}, g_{3}\right)+Q}{3 \wp^{\prime}\left(\xi ; g_{2}, g_{3}\right)}\right\}, \\
& v_{50}=\alpha^{2}-\beta-Q-\frac{2 P R\left[6 \wp\left(\xi ; g_{2}, g_{3}\right)+Q\right]^{2}}{9\left[\wp^{\prime}\left(\xi ; g_{2}, g_{3}\right)\right]^{2}} .
\end{aligned}
$$

Case 51. $g_{2}=(1 / 12) Q^{2}+P R, g_{3}=(1 / 216) Q\left(36 P R-Q^{2}\right)$, $F(\xi)=3 \wp^{\prime}\left(\xi ; g_{2}, g_{3}\right) / \sqrt{P}\left[6 \wp\left(\xi ; g_{2}, g_{3}\right)+Q\right]$,

$$
\begin{gathered}
u_{51}=e^{i \theta}\left\{ \pm 2 \sqrt{Q-\alpha-3 \alpha^{2}+3 \beta} \frac{3 \wp^{\prime}\left(\xi ; g_{2}, g_{3}\right)}{6 \wp\left(\xi ; g_{2}, g_{3}\right)+Q}\right\}, \\
v_{51}=\alpha^{2}-\beta-Q-\frac{18\left[\wp^{\prime}\left(\xi ; g_{2}, g_{3}\right)\right]^{2}}{\left[6 \wp\left(\xi ; g_{2}, g_{3}\right)+Q\right]^{2}} .
\end{gathered}
$$

Case 52. $R=5 Q^{2} / 36 P, g_{2}=2 Q^{2} / 9, g_{3}=Q^{3} / 54, F(\xi)=$ $Q \sqrt{-15 Q / 2 P} \wp\left(\xi ; g_{2}, g_{3}\right) /\left(3 \wp\left(\xi ; g_{2}, g_{3}\right)+Q\right)$,

$$
\begin{gathered}
u_{52}=e^{i \theta}\left\{ \pm 2 \sqrt{P\left(Q-\alpha-3 \alpha^{2}+3 \beta\right)}\right. \\
\left.\times \frac{Q \sqrt{-15 Q / 2 P} \wp\left(\xi ; g_{2}, g_{3}\right)}{3 \wp\left(\xi ; g_{2}, g_{3}\right)+Q}\right\}, \\
v_{52}=\alpha^{2}-\beta-Q+\frac{15 Q^{3} \wp^{2}\left(\xi ; g_{2}, g_{3}\right)}{\left[3 \wp\left(\xi ; g_{2}, g_{3}\right)+Q\right]^{2}} .
\end{gathered}
$$

It should be noted that any solution that can be expressed in terms of a Weierstrass-elliptic function can be also converted into a solution in terms of a Jacobian-elliptic function (for more details, see [53]). Consequently, Cases 47-52 are already covered in Cases 1-46. For example, using 1031.01 in [52] reveals that, with the $P, Q$, and $R$ values for Case $1, u_{1}$ and $u_{48}$ are identical and $v_{1}$ and $v_{48}$ are identical.

\section{New Soliton-Like Solutions of the Schrödinger-KdV Equation}

Some soliton-like solutions of (1) can be obtained in the limited case when the modulus $m \rightarrow 1$ (see Appendix C), as follows:

$$
\begin{aligned}
& u_{1}=e^{i \theta}\left\{ \pm 2 \sqrt{-2-\alpha-3 \alpha^{2}+3 \beta} \tanh \xi\right\}, \\
& v_{1}=\alpha^{2}-\beta+2 \operatorname{sech}^{2} \xi \\
& u_{3}=e^{i \theta}\left\{ \pm 2 \sqrt{-1+\alpha+3 \alpha^{2}-3 \beta} \operatorname{sech} \xi\right\}, \\
& v_{3}=\alpha^{2}-\beta-1+2 \operatorname{sech}^{2} \xi \\
& u_{5}=e^{i \theta}\left\{ \pm 2 \sqrt{-2-\alpha-3 \alpha^{2}+3 \beta} \operatorname{coth} \xi\right\}, \\
& v_{5}=\alpha^{2}-\beta-2 \operatorname{csch}^{2} \xi, \\
& u_{11}=e^{i \theta}\left\{ \pm 2 \sqrt{1-\alpha-3 \alpha^{2}+3 \beta} \operatorname{csch} \xi\right\}, \\
& v_{11}=\alpha^{2}-\beta-1-2 \operatorname{csch}^{2} \xi, \\
& u_{13}=e^{i \theta}\left\{ \pm \sqrt{\frac{-1-2 \alpha-6 \alpha^{2}+6 \beta}{2}}(\operatorname{coth} \xi \pm \operatorname{csch} \xi)\right\}, \\
& v_{13}=\frac{1}{2}\left\{2 \alpha^{2}-2 \beta+1-(\operatorname{coth} \xi \pm \operatorname{csch} \xi)^{2}\right\}, \\
& u_{16}=e^{i \theta}\left\{ \pm \sqrt{\frac{-1-2 \alpha-6 \alpha^{2}+6 \beta}{2}}(\tanh \xi \pm i \operatorname{sech} \xi)\right\}, \\
& v_{16}=\frac{1}{2}\left\{2 \alpha^{2}-2 \beta+1-(\tanh \xi \pm i \operatorname{sech} \xi)^{2}\right\},
\end{aligned}
$$




$$
\begin{aligned}
& u_{22}=e^{i \theta}\left\{ \pm \sqrt{\frac{-1-2 \alpha-6 \alpha^{2}+6 \beta}{2}} \frac{\tanh \xi}{1 \pm \operatorname{sech} \xi}\right\}, \quad \begin{array}{l}
\text { Here, it should be noted that each exact solution given in (67) } \\
\text { can be split into two solutions if one chooses the (+ve) and } \\
(-\mathrm{ve}) \text { signs, respectively, but they have not been calculated. }
\end{array} \\
& v_{22}=\frac{1}{2}\left\{2 \alpha^{2}-2 \beta+1-\left(\frac{\tanh \xi}{1 \pm \operatorname{sech} \xi}\right)^{2}\right\}, \\
& \begin{array}{l}
\text { Also, all the exact solutions given by (67) can be verified } \\
\text { by substitution. The main feature for some of these exact } \\
\text { solutions is the inclusion of the free parameters } Q, B, \text { and } C .
\end{array} \\
& u_{23}=e^{i \theta}\left\{ \pm 2 \sqrt{-1+\alpha+3 \alpha^{2}-3 \beta} \operatorname{sech} \xi\right\}, \\
& v_{23}=\alpha^{2}-\beta-1+2 \operatorname{sech}^{2} \xi,
\end{aligned}
$$

\section{New Trigonometric-Function Solutions of the Schrödinger-KdV Equation}$$
u_{26}=e^{i \theta}\left\{ \pm 2 \sqrt{\frac{Q\left(-Q+\alpha+3 \alpha^{2}-3 \beta\right)}{2}} \tanh \left(\sqrt{\frac{-Q}{2}} \xi\right)\right\} \text {, }
$$$$
v_{26}=\alpha^{2}-\beta-\operatorname{Qsech}^{2}\left(\sqrt{\frac{-Q}{2}} \xi\right) \text {, }
$$$$
u_{27}=e^{i \theta}\left\{ \pm 2 \sqrt{Q\left(-Q+\alpha+3 \alpha^{2}-3 \beta\right)} \operatorname{sech}(\sqrt{Q} \xi)\right\} \text {, }
$$$$
v_{27}=\alpha^{2}-\beta-Q\left[1-2 \operatorname{sech}^{2}(\sqrt{Q} \xi)\right],
$$$$
u_{30}=e^{i \theta}\left\{ \pm 2 \sqrt{2-\alpha-3 \alpha^{2}+3 \beta} \tanh \xi\right\} \text {, }
$$$$
v_{30}=\alpha^{2}-\beta-2\left(2-\operatorname{sech}^{2} \xi\right) \text {, }
$$$$
u_{31}=e^{i \theta}\left\{ \pm 2 \sqrt{3-\alpha-3 \alpha^{2}+3 \beta} \operatorname{sech} \xi \operatorname{csch} \xi\right\},
$$$$
v_{31}=\alpha^{2}-\beta-3-2(\operatorname{sech} \xi \operatorname{csch} \xi)^{2} \text {, }
$$$$
u_{34}=e^{i \theta}\left\{ \pm 4 \sqrt{-4+\alpha+3 \alpha^{2}-3 \beta} \frac{\operatorname{sech}^{2} \xi}{1+\tanh ^{2} \xi}\right\},
$$$$
v_{34}=\alpha^{2}-\beta-4+\frac{8 \operatorname{sech}^{4} \xi}{\left(1+\tanh ^{2} \xi\right)^{2}},
$$$$
u_{40}=e^{i \theta}\left\{ \pm \sqrt{\left(\frac{-1-2 \alpha-6 \alpha^{2}+6 \beta}{2}\right) \frac{1+\operatorname{sech} \xi}{1-\operatorname{sech} \xi}}\right\},
$$$$
v_{40}=\frac{1}{2}\left\{2 \alpha^{2}-2 \beta+1-\frac{1+\operatorname{sech} \xi}{1-\operatorname{sech} \xi}\right\} \text {, }
$$$$
u_{41}=e^{i \theta}\left\{ \pm \sqrt{\left(\frac{-1-2 \alpha-6 \alpha^{2}+6 \beta}{2}\right) \frac{1-\operatorname{sech} \xi}{1+\operatorname{sech} \xi}}\right\} \text {, }
$$$$
v_{41}=\frac{1}{2}\left\{2 \alpha^{2}-2 \beta+1-\frac{1-\operatorname{sech} \xi}{1+\operatorname{sech} \xi}\right\} \text {, }
$$$$
u_{43}=e^{i \theta}\left\{ \pm \sqrt{\frac{-1-2 \alpha-6 \alpha^{2}+6 \beta}{2}} \frac{B+\sqrt{B^{2}+C^{2}} \operatorname{sech} \xi}{B \tanh \xi+C \operatorname{sech} \xi}\right\} \text {, }
$$$$
v_{43}=\frac{1}{2}\left\{2 \alpha^{2}-2 \beta+1-\left(\frac{B+\sqrt{B^{2}+C^{2}} \operatorname{sech} \xi}{B \tanh \xi+C \operatorname{sech} \xi}\right)^{2}\right\} \text {. }
$$

Some trigonometric-function solutions of (1) can be obtained in the limited case when the modulus $m \rightarrow 0$. For example,

$$
\begin{aligned}
& u_{5}=e^{i \theta}\left\{ \pm 2 \sqrt{-1-\alpha-3 \alpha^{2}+3 \beta} \csc \xi\right\}, \\
& v_{5}=\alpha^{2}-\beta+1-2 \csc ^{2} \xi \\
& u_{6}=e^{i \theta}\left\{ \pm 2 \sqrt{-1-\alpha-3 \alpha^{2}+3 \beta} \sec \xi\right\}, \\
& v_{6}=\alpha^{2}-\beta+1-2 \sec ^{2} \xi \\
& u_{9}=e^{i \theta}\left\{ \pm 2 \sqrt{2-\alpha-3 \alpha^{2}+3 \beta} \tan \xi\right\}, \\
& v_{9}=\alpha^{2}-\beta-2 \sec ^{2} \xi \\
& u_{11}=e^{i \theta}\left\{ \pm 2 \sqrt{2-\alpha-3 \alpha^{2}+3 \beta} \cot \xi\right\}, \\
& v_{11}=\alpha^{2}-\beta-2 \csc \xi \\
& u_{13}=e^{i \theta}\left\{ \pm \sqrt{\frac{1-2 \alpha-6 \alpha^{2}+6 \beta}{2}}(\csc \xi \pm \cot \xi)\right\}, \\
& v_{13}=\frac{1}{2}\left\{2 \alpha^{2}-2 \beta-1-(\csc \xi \pm \cot \xi)^{2}\right\}, \\
& v_{24}=\frac{1}{2}\left\{2 \alpha^{2}-2 \beta-1+(\csc \xi \pm \cot \xi)^{2}\right\}, \\
& u_{14}=e^{i \theta}\left\{ \pm \sqrt{\frac{1-2 \alpha-6 \alpha^{2}+6 \beta}{2}}(\sec \xi \pm \tan \xi)\right\}, \\
& v_{34}=e^{i \theta}\left\{ \pm \sqrt{\frac{1-2 \alpha-6 \alpha^{2}+6 \beta}{2}}(\csc \xi \pm \cot \xi)\right\}, \\
& u_{24}=e^{i \theta}\left\{ \pm \sqrt{\left.\frac{1-2 \alpha-6 \alpha^{2}+6 \beta}{2}(\csc \xi \pm \cot \xi)\right\}},\right.
\end{aligned}
$$




$$
\begin{aligned}
& v_{36}=\frac{1}{2}\left\{2 \alpha^{2}-2 \beta-1-(\csc \xi \pm \cot \xi)^{2}\right\}, \\
& u_{37}=e^{i \theta}\left\{ \pm \sqrt{\frac{1-2 \alpha-6 \alpha^{2}+6 \beta}{2}}(\sec \xi \pm \tan \xi)\right\}, \\
& v_{37}=\frac{1}{2}\left\{2 \alpha^{2}-2 \beta-1-(\sec \xi \pm \tan \xi)^{2}\right\} \text {, } \\
& u_{42}=e^{i \theta}\left\{ \pm \sqrt{\frac{1-2 \alpha-6 \alpha^{2}+6 \beta}{2}} \frac{\sqrt{B^{2}-C^{2}}+B \sin \xi}{B \cos \xi+C}\right\} \text {, } \\
& v_{42}=\frac{1}{2}\left\{2 \alpha^{2}-2 \beta-1-\left(\frac{\sqrt{B^{2}-C^{2}}+B \sin \xi}{B \cos \xi+C}\right)^{2}\right\} \text {, } \\
& u_{43}=e^{i \theta}\left\{ \pm \sqrt{\frac{1-2 \alpha-6 \alpha^{2}+6 \beta}{2}} \frac{\sqrt{B^{2}-C^{2}}+B \cos \xi}{B \sin \xi+C}\right\} \text {, } \\
& v_{43}=\frac{1}{2}\left\{2 \alpha^{2}-2 \beta-1-\left(\frac{\sqrt{B^{2}-C^{2}}+B \cos \xi}{B \sin \xi+C}\right)^{2}\right\} \text {, } \\
& u_{44}=e^{i \theta}\left\{ \pm \sqrt{-1-\alpha-3 \alpha^{2}+3 \beta} \frac{2 \sqrt{B^{2}+C^{2}}}{B \sin \xi+C \cos \xi}\right\} \text {, } \\
& v_{44}=\alpha^{2}-\beta+1-\frac{2\left(B^{2}+C^{2}\right)}{(B \sin \xi+C \cos \xi)^{2}} \text {. }
\end{aligned}
$$

Here, we note also that each trigonometric-function solution obtained in this section can split into two solutions if we choose the (+ve) and (-ve) signs, respectively. Besides, all these solutions can be verified by direct substitution. Also, the main feature for some of these exact solutions is the inclusion of the free parameters $Q, B$, and $C$.

\section{Conclusion}

In this paper, the $F$-expansion method has been applied to construct 52 types of exact solution of the the Schrödinger$\mathrm{KdV}$ equation. The main advantage of this method over other methods is that it possesses all types of exact solution, including those of Jacobian-elliptic and Weierstrasselliptic functions. Moreover, the soliton-like solutions and trigonometric-function solutions have been also obtained as the modulus $m$ of Jacobi-elliptic function approaches to 1 and 0 . It can be said that the results in this paper provide good supplements to the existing literature and are useful for describing certain nonlinear phenomena. This method can be applied to many other nonlinear evolution equations. Finally, it is worthwhile to mention that the proposed method is also a straightforward, short, promising, and powerful method for other nonlinear evolution equations in mathematical physics.

\section{Appendices}

\section{A. Relations between Values of $(P, Q, R)$ and Corresponding $F(\xi)$ in (7)}

Relations between values of $(P, Q, R)$ and corresponding $F(\xi)$ in (7), where $A, B$, and $C$ are arbitrary constants and $m_{1}=$ $\sqrt{1-m^{2}}$. As shown in Table 1 .

\section{B. The Weierstrass-Elliptic Function Solutions for $(7)$}

The Weierstrass-elliptic function solutions for (7), where $D=(1 / 2)\left(-5 Q \pm \sqrt{9 Q^{2}-36 P R}\right)$ and $\wp^{\prime}\left(\xi ; g_{2}, g_{3}\right)=$ $d \wp\left(\xi ; g_{2}, g_{3}\right) / d \xi$. As shown in Table 2 .

\section{Relations between Jacobian-Elliptic Functions and Hyperbolic Functions}

The Jacobian-elliptic functions degenerate into hyperbolic functions when $m \rightarrow 1$ as follows:

$$
\begin{aligned}
& \operatorname{sn} \xi \longrightarrow \tanh \xi, \quad c n \xi \longrightarrow \operatorname{sech} \xi, \quad d n \xi \longrightarrow \operatorname{sech} \xi, \\
& s c \xi \longrightarrow \sinh \xi, \quad s d \xi \longrightarrow \sinh \xi, \quad c d \xi \longrightarrow 1, \\
& n s \xi \longrightarrow \operatorname{coth} \xi, \quad n c \xi \longrightarrow \cosh \xi, \quad n d \xi \longrightarrow \cosh \xi \text {, } \\
& \operatorname{cs} \xi \longrightarrow \operatorname{csch} \xi, \quad d s \xi \longrightarrow \operatorname{csch} \xi, \quad d c \xi \longrightarrow 1 \text {. }
\end{aligned}
$$

The Jacobian-elliptic functions degenerate into trigonometric functions when $m \rightarrow 0$ as follows:

$$
\begin{array}{lll}
\operatorname{sn} \xi \longrightarrow \sin \xi, & c n \xi \longrightarrow \cos \xi, & d n \xi \longrightarrow 1, \\
s c \xi \longrightarrow \tan \xi, & s d \xi \longrightarrow \sin \xi, & c d \xi \longrightarrow \cos \xi, \\
n s \xi \longrightarrow \csc \xi, & n c \xi \longrightarrow \sec \xi, & n d \xi \longrightarrow 1, \\
c s \xi \longrightarrow \cot \xi, & d s \xi \longrightarrow \csc \xi, & d c \xi \longrightarrow \sec \xi .
\end{array}
$$

\section{Some Trigonometric and Hyperbolic Identities}

Consider the following:

$$
\begin{gathered}
\operatorname{coth} \theta-\operatorname{csch} \theta=\tanh \frac{\theta}{2}, \quad \csc \theta-\cot \theta=\tan \frac{\theta}{2}, \\
\operatorname{coth} \theta+\operatorname{csch} \theta=\operatorname{coth} \frac{\theta}{2}, \quad \csc \theta+\cot \theta=\cot \frac{\theta}{2}, \\
\tanh \theta+i \operatorname{sech} \theta=\tanh \left[\frac{1}{2}\left(\theta+\frac{i \pi}{2}\right)\right], \\
\sec \theta+\tan \theta=\tan \left[\frac{1}{2}\left(\theta+\frac{\pi}{2}\right)\right], \\
\tanh \theta-i \operatorname{sech} \theta=\operatorname{coth}\left[\frac{1}{2}\left(\theta+\frac{i \pi}{2}\right)\right], \\
\sec \theta-\tan \theta=\cot \left[\frac{1}{2}\left(\theta+\frac{\pi}{2}\right)\right] .
\end{gathered}
$$




\begin{tabular}{|c|c|c|c|c|}
\hline Case & $P$ & $Q$ & $R$ & $F(\xi)$ \\
\hline 1 & $m^{2}$ & $-\left(1+m^{2}\right)$ & 1 & $\operatorname{sn} \xi$ \\
\hline 2 & $m^{2}$ & $-\left(1+m^{2}\right)$ & 1 & $c d \xi=\frac{c n \xi}{d n \xi}$ \\
\hline 3 & $-m^{2}$ & $2 m^{2}-1$ & $1-m^{2}$ & $c n \xi$ \\
\hline 4 & -1 & $2-m^{2}$ & $m^{2}-1$ & $d n \xi$ \\
\hline 5 & 1 & $-\left(1+m^{2}\right)$ & $m^{2}$ & $n s \xi=(s n \xi)^{-1}$ \\
\hline 6 & 1 & $-\left(1+m^{2}\right)$ & $m^{2}$ & $d c \xi=\frac{d n \xi}{c n \xi}$ \\
\hline 7 & $1-m^{2}$ & $2 m^{2}-1$ & $-m^{2}$ & $n c \xi=(c n \xi)^{-1}$ \\
\hline 8 & $m^{2}-1$ & $2-m^{2}$ & -1 & $n d \xi=(d n \xi)^{-1}$ \\
\hline 9 & $1-m^{2}$ & $2-m^{2}$ & 1 & $s c \xi=\frac{s n \xi}{c n \xi}$ \\
\hline 10 & $-m^{2}\left(1-m^{2}\right)$ & $2 m^{2}-1$ & 1 & $s d \xi=\frac{s n \xi}{d n \xi}$ \\
\hline 11 & 1 & $2-m^{2}$ & $1-m^{2}$ & $c s \xi=\frac{c n \xi}{s n \xi}$ \\
\hline 12 & 1 & $2 m^{2}-1$ & $-m^{2}\left(1-m^{2}\right)$ & $d s \xi=\frac{d n \xi}{s n \xi}$ \\
\hline 13 & $\frac{1}{4}$ & $\frac{1-2 m^{2}}{2}$ & $\frac{1}{4}$ & $n s \xi \pm c s \xi$ \\
\hline 14 & $\frac{1-m^{2}}{4}$ & $\frac{1+m^{2}}{2}$ & $\frac{1-m^{2}}{4}$ & $n c \xi \pm s c \xi$ \\
\hline 15 & $\frac{1}{4}$ & $\frac{m^{2}-2}{2}$ & $\frac{m^{2}}{4}$ & $n s \xi \pm d s \xi$ \\
\hline 16 & $\frac{m^{2}}{4}$ & $\frac{m^{2}-2}{2}$ & $\frac{m^{2}}{4}$ & $s n \xi \pm i c n \xi$ \\
\hline 17 & $\frac{m^{2}}{4}$ & $\frac{m^{2}-2}{2}$ & $\frac{m^{2}}{4}$ & $\sqrt{1-m^{2}} s d \xi \pm c d \xi$ \\
\hline 18 & $\frac{1}{4}$ & $\frac{1-m^{2}}{2}$ & $\frac{1}{4}$ & $m c d \xi \pm i \sqrt{1-m^{2}} n d \xi$ \\
\hline 19 & $\frac{1}{4}$ & $\frac{1-2 m^{2}}{2}$ & $\frac{1}{4}$ & $m s n \xi \pm i d n \xi$ \\
\hline 20 & $\frac{1}{4}$ & $\frac{1-m^{2}}{2}$ & $\frac{1}{4}$ & $\sqrt{1-m^{2}} s c \xi \pm d c \xi$ \\
\hline 21 & $\frac{m^{2}-1}{4}$ & $\frac{m^{2}+1}{2}$ & $\frac{m^{2}-1}{4}$ & $m s d \xi \pm n d \xi$ \\
\hline 22 & $\frac{m^{2}}{4}$ & $\frac{m^{2}-2}{2}$ & $\frac{1}{4}$ & $\frac{s n \xi}{1 \pm d n \xi}$ \\
\hline 23 & $-\frac{1}{4}$ & $\frac{m^{2}+1}{2}$ & $\frac{\left(1-m^{2}\right)^{2}}{4}$ & $m c n \xi \pm d n \xi$ \\
\hline 24 & $\frac{\left(1-m^{2}\right)^{2}}{4}$ & $\frac{m^{2}+1}{2}$ & $\frac{1}{4}$ & $d s \xi \pm c s \xi$ \\
\hline 25 & $\frac{m^{4}\left(1-m^{2}\right)}{2\left(2-m^{2}\right)}$ & $\frac{2\left(1-m^{2}\right)}{m^{2}-2}$ & $\frac{1-m^{2}}{2\left(2-m^{2}\right)}$ & $d c \xi \pm \sqrt{1-m^{2}} n c \xi$ \\
\hline 26 & $P>0$ & $Q<0$ & $\frac{m^{2} Q^{2}}{\left(1+m^{2}\right)^{2} P}$ & $\frac{-m^{2} Q}{\left(1+m^{2}\right) P} s n\left(\sqrt{\frac{-Q}{1+m}}\right.$ \\
\hline
\end{tabular}


TABle 1: Continued.

\begin{tabular}{|c|c|c|c|c|}
\hline Case & $P$ & Q & $R$ & $F(\xi)$ \\
\hline 27 & $P<0$ & $Q>0$ & $\frac{\left(1-m^{2}\right) Q^{2}}{\left(m^{2}-2\right)^{2} P}$ & $\sqrt{\frac{-Q}{\left(2-m^{2}\right) P}} d n\left(\sqrt{\frac{Q}{2-m^{2}}} \xi\right)$ \\
\hline 28 & $P<0$ & $Q>0$ & $\frac{m^{2}\left(m^{2}-1\right) Q^{2}}{\left(2 m^{2}-1\right)^{2} P}$ & $\sqrt{-\frac{m^{2} Q}{\left(2 m^{2}-1\right) P}} c n\left(\sqrt{\frac{Q}{2 m^{2}-1}} \xi\right)$ \\
\hline 29 & 1 & $2-4 m^{2}$ & 1 & $\frac{s n \xi d n \xi}{c n \xi}$ \\
\hline 30 & $m^{4}$ & 2 & 1 & $\frac{s n \xi c n \xi}{d n \xi}$ \\
\hline 31 & 1 & $m^{2}+2$ & $1-2 m^{2}+m^{4}$ & $\frac{d n \xi c n \xi}{s n \xi}$ \\
\hline \multirow{2}{*}{32} & $A^{2}(m-1)^{2}$ & $\underline{m^{2}+1}+3 m$ & $\underline{(m-1)^{2}}$ & $d n \xi c n \xi$ \\
\hline & 4 & $2+J m$ & $\overline{4 A^{2}}$ & $\overline{A(1+s n \xi)(1+m s n \xi)}$ \\
\hline \multirow{2}{*}{33} & $\underline{A}^{2}(m+1)^{2}$ & $\underline{m^{2}+1}-3 m$ & $\underline{(m+1)^{2}}$ & $\frac{d n \xi c n \xi}{}$ \\
\hline & 4 & $2-5 m$ & $4 A^{2}$ & $\overline{A(1+s n \xi)(1-m s n \xi)}$ \\
\hline 34 & $-\frac{4}{m}$ & $6 m-m^{2}-1$ & $-2 m^{3}+m^{4}+m^{2}$ & $\frac{m c n \xi d n \xi}{m s n^{2} \xi+1}$ \\
\hline 35 & $\frac{4}{m}$ & $-6 m-m^{2}-1$ & $2 m^{3}+m^{4}+m^{2}$ & $\frac{m c n \xi d n \xi}{m s n^{2} \xi-1}$ \\
\hline \multirow{2}{*}{36} & 1 & $1-2 m^{2}$ & $\underline{1}$ & $s n \xi$ \\
\hline & $\overline{4}$ & 2 & $\overline{4}$ & $\overline{1 \pm c n \xi}$ \\
\hline \multirow{2}{*}{37} & $\underline{1-m^{2}}$ & $\underline{1+m^{2}}$ & $1-m^{2}$ & $c n \xi$ \\
\hline & 4 & 2 & 4 & $\overline{1 \pm \operatorname{sn\xi }}$ \\
\hline 38 & $4 m_{1}$ & $2+6 m_{1}-m^{2}$ & $2+2 m_{1}-m^{2}$ & $\frac{m^{2} s n \xi c n \xi}{m_{1}-d n^{2} \xi}$ \\
\hline 39 & $-4 m_{1}$ & $2-6 m_{1}-m^{2}$ & $2-2 m_{1}-m^{2}$ & $-\frac{m^{2} s n \xi c n \xi}{m_{1}+d n^{2} \xi}$ \\
\hline \multirow{2}{*}{40} & $2-m^{2}-2 m_{1}$ & $\underline{m^{2}}-1-3 m$ & $2-m^{2}-2 m_{1}$ & $m^{2} \operatorname{sn} \xi c n \xi$ \\
\hline & 4 & 2 & 4 & $s n^{2} \xi+\left(1+m_{1}\right) d n \xi-1-m_{1}$ \\
\hline 41 & $\underline{2-m^{2}+2 m_{1}}$ & $\underline{m^{2}}-1+3 m$ & $\underline{2-m^{2}+2 m_{1}}$ & $m^{2} \operatorname{sn} \xi c n \xi$ \\
\hline & 4 & 2 & 4 & $s n^{2} \xi+\left(-1+m_{1}\right) d n \xi-1+m_{1}$ \\
\hline \multirow{2}{*}{42} & $C^{2} m^{4}-\left(B^{2}+C^{2}\right) m^{2}+B^{2}$ & $\underline{m^{2}+1}$ & $m^{2}-1$ & $\sqrt{\left(\left(B^{2}-C^{2}\right) /\left(B^{2}-C^{2} m^{2}\right)\right)}+s n \xi$ \\
\hline & $\overline{4}$ & 2 & $\overline{4\left(C^{2} m^{2}-B^{2}\right)}$ & $\overline{B c n \xi+C d n \xi}$ \\
\hline \multirow{2}{*}{43} & $B^{2}+C^{2} m^{2}$ & $\frac{1}{-}-m^{2}$ & $\frac{1}{1\left(0^{2}+2\right)^{2}}$ & $\sqrt{\left(\left(C^{2} m^{2}+B^{2}-C^{2}\right) /\left(B^{2}+C^{2} m^{2}\right)\right)}+c n \xi$ \\
\hline & 4 & $\overline{2}^{-m}$ & $\overline{4\left(C^{2} m^{2}+B^{2}\right)}$ & $\overline{B s n \xi+C d n \xi}$ \\
\hline \multirow{2}{*}{44} & $B^{2}+C^{2}$ & $\underline{m^{2}}-1$ & $m^{4}$ & $\sqrt{\left(\left(B^{2}+C^{2}-C^{2} m^{2}\right) /\left(B^{2}+C^{2}\right)\right)}+d n \xi$ \\
\hline & 4 & $\overline{2}^{-1}$ & $\overline{4\left(C^{2}+B^{2}\right)}$ & $B s n \xi+C c n \xi$ \\
\hline 45 & $-\left(m^{2}+2 m+1\right) B^{2}$ & $2 m^{2}+2$ & $\frac{2 m-m^{2}-1}{B^{2}}$ & $\frac{m s n^{2} \xi-1}{B\left(m s n^{2} \xi+1\right)}$ \\
\hline 46 & $-\left(m^{2}-2 m+1\right) B^{2}$ & $2 m^{2}+2$ & $-\frac{2 m+m^{2}+1}{B^{2}}$ & $\frac{m s n^{2} \xi+1}{B\left(m s n^{2} \xi-1\right)}$ \\
\hline
\end{tabular}


TABLE 2

\begin{tabular}{|c|c|c|c|}
\hline Case & $g_{2}$ & $g_{3}$ & $F(\xi)$ \\
\hline 47 & $\frac{4}{3}\left(Q^{2}-3 P R\right)$ & $\frac{4 Q}{27}\left(-2 Q^{2}+9 P R\right)$ & $\sqrt{\frac{1}{P}\left(\wp\left(\xi ; g_{2}, g_{3}\right)-\frac{1}{3} Q\right)}$ \\
\hline 48 & $\frac{4}{3}\left(Q^{2}-3 P R\right)$ & $\frac{4 Q}{27}\left(-2 Q^{2}+9 P R\right)$ & $\sqrt{\frac{3 R}{3 \wp\left(\xi ; g_{2}, g_{3}\right)-Q}}$ \\
\hline 49 & $5 Q D+4 Q^{2}+33 P Q R$ & $21 Q^{2} D-63 P R D+20 Q^{3}-27 P Q R$ & $\sqrt{12 R \wp\left(\xi ; g_{2}, g_{3}\right)+2 R(2 Q+D)}$ \\
\hline 49 & 12 & $\begin{array}{l}216 \\
\end{array}$ & $12 \wp\left(\xi ; g_{2}, g_{3}\right)+D$ \\
\hline 50 & $\frac{1}{12} Q^{2}+P R$ & $\frac{1}{216} Q\left(36 P R-Q^{2}\right)$ & $\frac{\sqrt{R}\left[6 \wp\left(\xi ; g_{2}, g_{3}\right)+Q\right]}{3 \wp^{\prime}\left(\xi ; g_{2}, g_{3}\right)}$ \\
\hline 51 & $\frac{1}{12} Q^{2}+P R$ & $\frac{1}{216} Q\left(36 P R-Q^{2}\right)$ & $\frac{3 \wp^{\prime}\left(\xi ; g_{2}, g_{3}\right)}{\sqrt{P}\left[6 \wp\left(\xi ; g_{2}, g_{3}\right)+Q\right]}$ \\
\hline 52 & $\frac{2 Q^{2}}{9}$ & $\frac{Q^{3}}{54}$ & $\frac{Q \sqrt{-15 Q / 2 P} \wp\left(\xi ; g_{2}, g_{3}\right)}{3 \wp\left(\xi ; g_{2}, g_{3}\right)+Q}, R=\frac{5 Q^{2}}{36 P}$ \\
\hline
\end{tabular}

\section{Conflict of Interests}

The authors declare that there is no conflict of interests regarding the publication of this paper.

\section{References}

[1] M. J. Ablowitz and P. A. Clarkson, Solitons, Nonlinear Evolution Equations and Inverse Scattering, Cambridge University Press, Cambridge, UK, 1991.

[2] M. Wadati, "Wave propagation in nonlinear lattice. I," Journal of the Physical Society of Japan, vol. 38, no. 3, pp. 673-680, 1975.

[3] V. B. Matveev and M. A. Salle, Darboux Transformation and Solitons, Springer Series in Nonlinear Dynamics, Springer, Berlin, Germany, 1991.

[4] R. Hirota, "Exact solution of the korteweg-de Vries equation for multiple collisions of solitons," Physical Review Letters, vol. 27, no. 18, pp. 1192-1194, 1971.

[5] R. Hirota, The Direct Method in Soliton Theory, Cambridge University Press, Cambridge, UK, 2004.

[6] J. Weiss, M. Tabor, and G. Carnevale, “The painlevé property for partial differential equations," Journal of Mathematical Physics, vol. 24, no. 3, pp. 522-526, 1982.

[7] J.-H. He, "Homotopy perturbation method for bifurcation of nonlinear problems," International Journal of Nonlinear Sciences and Numerical Simulation, vol. 6, no. 2, pp. 207-208, 2005.

[8] J.-H. He, "Application of homotopy perturbation method to nonlinear wave equations," Chaos, Solitons and Fractals, vol. 26, no. 3, pp. 695-700, 2005.

[9] M. Wang, "Exact solutions for a compound KdV-Burgers equation," Physics Letters A, vol. 213, no. 5-6, pp. 279-287, 1996.

[10] J.-H. He, "Variational principles for some nonlinear partial differential equations with variable coefficients," Chaos, Solitons and Fractals, vol. 19, no. 4, pp. 847-851, 2004.
[11] J.-H. He, "Variational approach to (2+1)-dimensional dispersive long water equations," Physics Letters A, vol. 335, no. 2-3, pp. 182-184, 2005.

[12] H.-M. Liu, "Variational approach to nonlinear electrochemical system," International Journal of Nonlinear Sciences and Numerical Simulation, vol. 5, no. 1, pp. 95-96, 2004.

[13] W. Malfliet, "Solitary wave solutions of nonlinear wave equations," American Journal of Physics, vol. 60, no. 7, pp. 650-654, 1992.

[14] E. J. Parkes and B. R. Duffy, "Travelling solitary wave solutions to a compound KdV-Burgers equation," Physics Letters A, vol. 229, no. 4, pp. 217-220, 1997.

[15] E. Fan, "Extended tanh-function method and its applications to nonlinear equations," Physics Letters A, vol. 277, no. 4-5, pp. 212-218, 2000.

[16] Z. Yan and H. Zhang, "New explicit solitary wave solutions and periodic wave solutions for Whitham-Broer-Kaup equation in shallow water," Physics Letters A, vol. 285, no. 5-6, pp. 355-362, 2001.

[17] A.-M. Wazwaz, "An analytic study of compactons structures in a class of nonlinear dispersive equations," Mathematics and Computers in Simulation, vol. 63, no. 1, pp. 35-44, 2003.

[18] M. Wang, X. Li, and J. Zhang, “The $\left(G^{\prime} / G\right)$-expansion method and travelling wave solutions of nonlinear evolution equations in mathematical physics," Physics Letters A, vol. 372, no. 4, pp. 417-423, 2008.

[19] G. Ebadi and A. Biswas, "The $\left(G^{\prime} / G\right)$ method and topological soliton solution of the $\mathrm{K}(\mathrm{m}, \mathrm{n})$ equation," Communications in Nonlinear Science and Numerical Simulation, vol. 16, no. 6, pp. 2377-2382, 2011.

[20] C.-S. Liu, "Trial equation method and its applications to nonlinear evolution equations," Acta Physica Sinica, vol. 54, no. 6, pp. 2505-2509, 2005. 
[21] C.-S. Liu, "A new trial equation method and its applications," Communications in Theoretical Physics, vol. 45, no. 3, pp. 395397, 2006.

[22] C.-S. Liu, "Trial equation method to nonlinear evolution equations with rank inhomogeneous: mathematical discussions and its applications," Communications in Theoretical Physics, vol. 45, no. 2, pp. 219-223, 2006.

[23] C. S. Liu, "Using trial equation method to solve the exact solutions for two kinds of KdV equations with variable coefficients," Acta Physica Sinica, vol. 54, no. 10, pp. 4506-4510, 2005.

[24] C.-S. Liu, "Applications of complete discrimination system for polynomial for classifications of traveling wave solutions to nonlinear differential equations," Computer Physics Communications, vol. 181, no. 2, pp. 317-324, 2010.

[25] Y. Gurefe, A. Sonmezoglu, and E. Misirli, "Application of the trial equation method for solving some nonlinear evolution equations arising in mathematical physics," Pramana, vol. 77, no. 6, pp. 1023-1029, 2011.

[26] Y. Pandir, Y. Gurefe, U. Kadak, and E. Misirli, "Classification of exact solutions for some nonlinear partial differential equations with generalized evolution," Abstract and Applied Analysis, vol. 2012, Article ID 478531, 16 pages, 2012.

[27] Y. Gurefe, E. Misirli, A. Sonmezoglu, and M. Ekici, "Extended trial equation method to generalized nonlinear partial differential equations," Applied Mathematics and Computation, vol. 219, no. 10, pp. 5253-5260, 2013.

[28] Y. Gurefe, E. Misirli, Y. Pandir, A. Sonmezoglu, and M. Ekici, "New exact solutions of the Davey-Stewartson equation withpower-law nonlinearity," Bulletin of the Malaysian Mathematical Sciences Society. Accepted.

[29] F. Kangalgil and F. Ayaz, "New exact travelling wave solutions for the Ostrovsky equation," Physics Letters A, vol. 372, no. 11, pp. 1831-1835, 2008.

[30] S. Liu, Z. Fu, S. Liu, and Q. Zhao, "Jacobi elliptic function expansion method and periodic wave solutions of nonlinear wave equations," Physics Letters A, vol. 289, no. 1-2, pp. 69-74, 2001.

[31] Z. Fu, S. Liu, S. Liu, and Q. Zhao, "New Jacobi elliptic function expansion and new periodic solutions of nonlinear wave equations," Physics Letters A, vol. 290, no. 1-2, pp. 72-76, 2001.

[32] G.-T. Liu and T.-Y. Fan, "New applications of developed Jacobi elliptic function expansion methods," Physics Letters A, vol. 345, no. 1-3, pp. 161-166, 2005.

[33] H. Zhang, "Extended Jacobi elliptic function expansion method and its applications," Communications in Nonlinear Science and Numerical Simulation, vol. 12, no. 5, pp. 627-635, 2007.

[34] H.-T. Chen and H.-Q. Zhang, "New double periodic and multiple soliton solutions of the generalized (2+1)-dimensional Boussinesq equation," Chaos, Solitons and Fractals, vol. 20, no. 4, pp. 765-769, 2004.

[35] D. Zhang, "Doubly periodic solutions of the modified Kawahara equation," Chaos, Solitons and Fractals, vol. 25, no. 5, pp. 11551160, 2005.

[36] Q. Liu and J.-M. Zhu, "Exact Jacobian elliptic function solutions and hyperbolic function solutions for Sawada-Kotere equation with variable coefficient," Physics Letters A, vol. 352, no. 3, pp. 233-238, 2006.

[37] X. Zhao, H. Zhi, and H. Zhang, "Improved Jacobi-function method with symbolic computation to construct new doubleperiodic solutions for the generalized Ito system," Chaos, Solitons and Fractals, vol. 28, no. 1, pp. 112-126, 2006.
[38] A. Ebaid and E. H. Aly, "Exact solutions for the transformed reduced Ostrovsky equation via the F-expansion method in terms of Weierstrass-elliptic and Jacobian-elliptic functions," Wave Motion, vol. 49, no. 2, pp. 296-308, 2012.

[39] J.-H. He and X.-H. Wu, "Exp-function method for nonlinear wave equations," Chaos, Solitons and Fractals, vol. 30, no. 3, pp. 700-708, 2006.

[40] A. Ebaid, "Exact solitary wave solutions for some nonlinear evolution equations via Exp-function method," Physics Letters A, vol. 365, no. 3, pp. 213-219, 2007.

[41] A. Ebaid, "Exact solutions for the generalized Klein-Gordon equation via a transformation and Exp-function method and comparison with Adomian's method," Journal of Computational and Applied Mathematics, vol. 223, no. 1, pp. 278-290, 2009.

[42] C. Köroǧlu and T. Öziş, "A novel traveling wave solution for Ostrovsky equation using Exp-function method," Computers and Mathematics with Applications, vol. 58, no. 11-12, pp. 21422146, 2009.

[43] Z. Yan, "An improved algebra method and its applications in nonlinear wave equations," MM Research Preprints, no. 22, pp. 264-274, 2003.

[44] A.-M. Wazwaz, "The tanh method for travelling wave solutions to the Zhiber-Shabat equation and other related equations," Communications in Nonlinear Science and Numerical Simulation, vol. 13, no. 3, pp. 584-592, 2008.

[45] T. Yoshinaga, M. Wakamiya, and T. Kakutani, "Recurrence and chaotic behavior resulting from nonlinear interaction between long and short waves," Physics of Fluids A, vol. 3, no. 1, pp. 83-89, 1991.

[46] N. N. Rao, "Nonlinear wave modulations in plasmas," Pramana, vol. 49, no. 1, pp. 109-127, 1997.

[47] S. V. Singh, N. N. Rao, and P. K. Shukla, "Nonlinearly coupled Langmuir and dust-acoustic waves in a dusty plasma," Journal of Plasma Physics, vol. 60, no. 3, pp. 551-567, 1998.

[48] S. Liu, Z. Fu, S. Liu, and Q. Zhao, "Jacobi elliptic function expansion method and periodic wave solutions of nonlinear wave equations," Physics Letters A, vol. 289, no. 1-2, pp. 69-74, 2001.

[49] D. Kaya and S. M. El-Sayed, "On the solution of the coupled Schrödinger-KdV equation by the decomposition method," Physics Letters A, vol. 313, no. 1-2, pp. 82-88, 2003.

[50] C.-L. Bai and H. Zhao, "Generalized method to construct the solitonic solutions to (3+1)-dimensional nonlinear equation," Physics Letters A, vol. 354, no. 5-6, pp. 428-436, 2006.

[51] B. Salim Bahrami, H. Abdollahzadeh, I. M. Berijani, D. D. Ganji, and M. Abdollahzadeh, "Exact travelling solutions for some nonlinear physical models by $\left(G^{\prime} / G\right)$-expansion method," Pramana, vol. 77, no. 2, pp. 263-275, 2011.

[52] P. F. Byrd and M. D. Friedman, Handbook of Elliptic Integrals for Engineers and Scientists, Springer, New York, NY, USA, 1971.

[53] A. Ebaid and S. M. Khaled, "New types of exact solutions for nonlinear Schrödinger equation with cubic nonlinearity," Journal of Computational and Applied Mathematics, vol. 235, no. 8, pp. 1984-1992, 2011. 


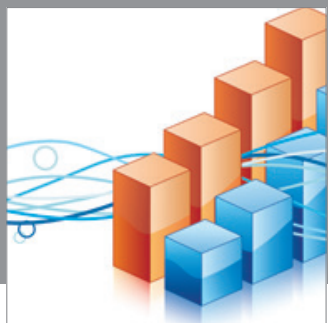

Advances in

Operations Research

mansans

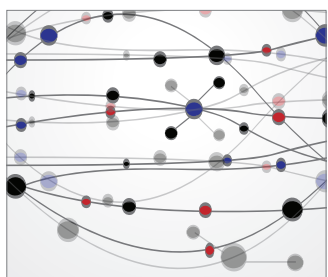

The Scientific World Journal
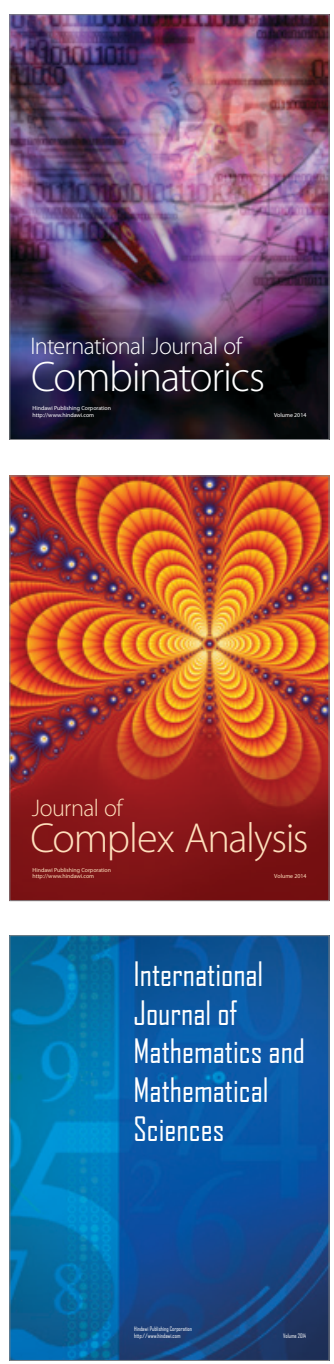
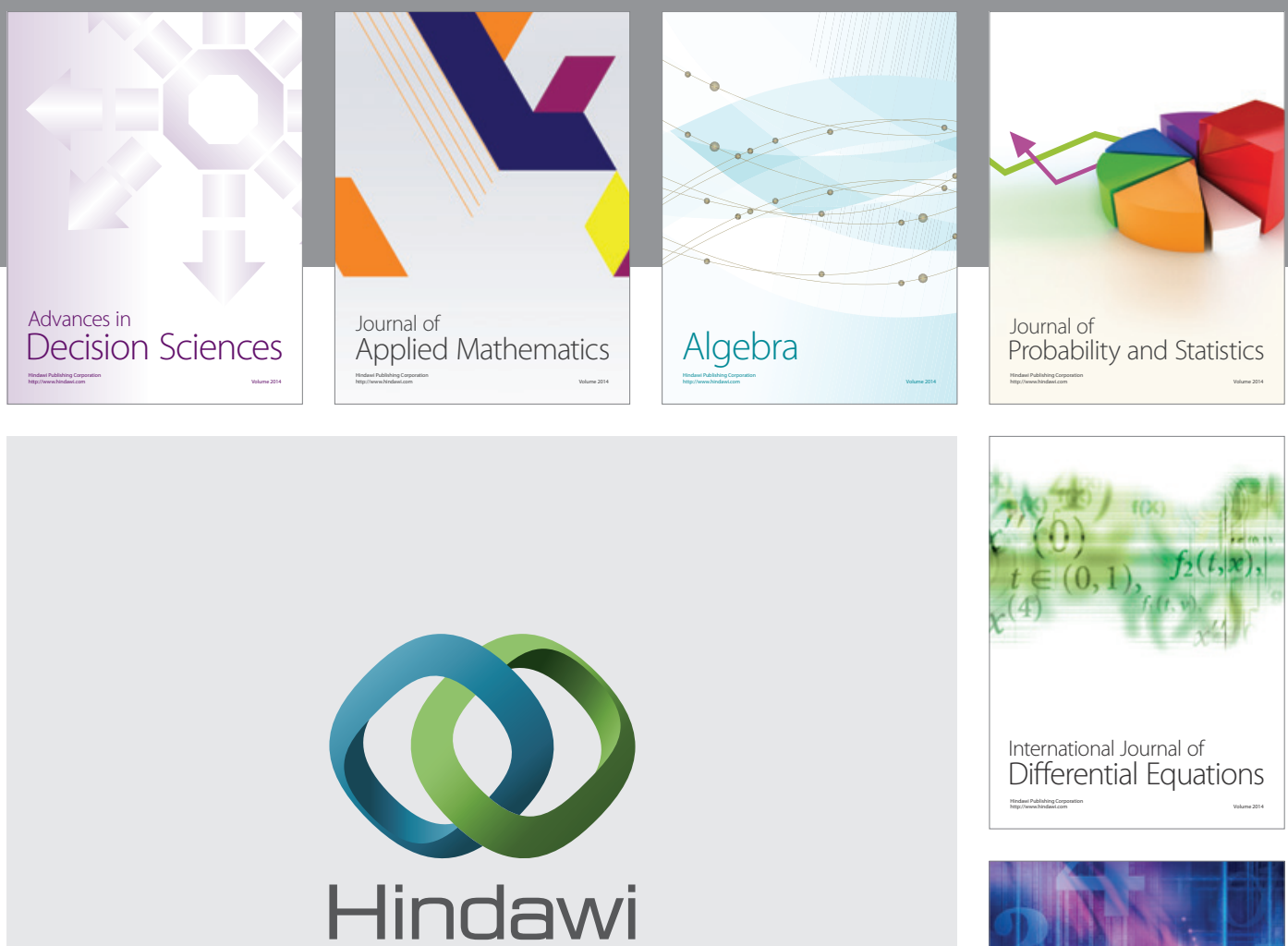

Submit your manuscripts at http://www.hindawi.com
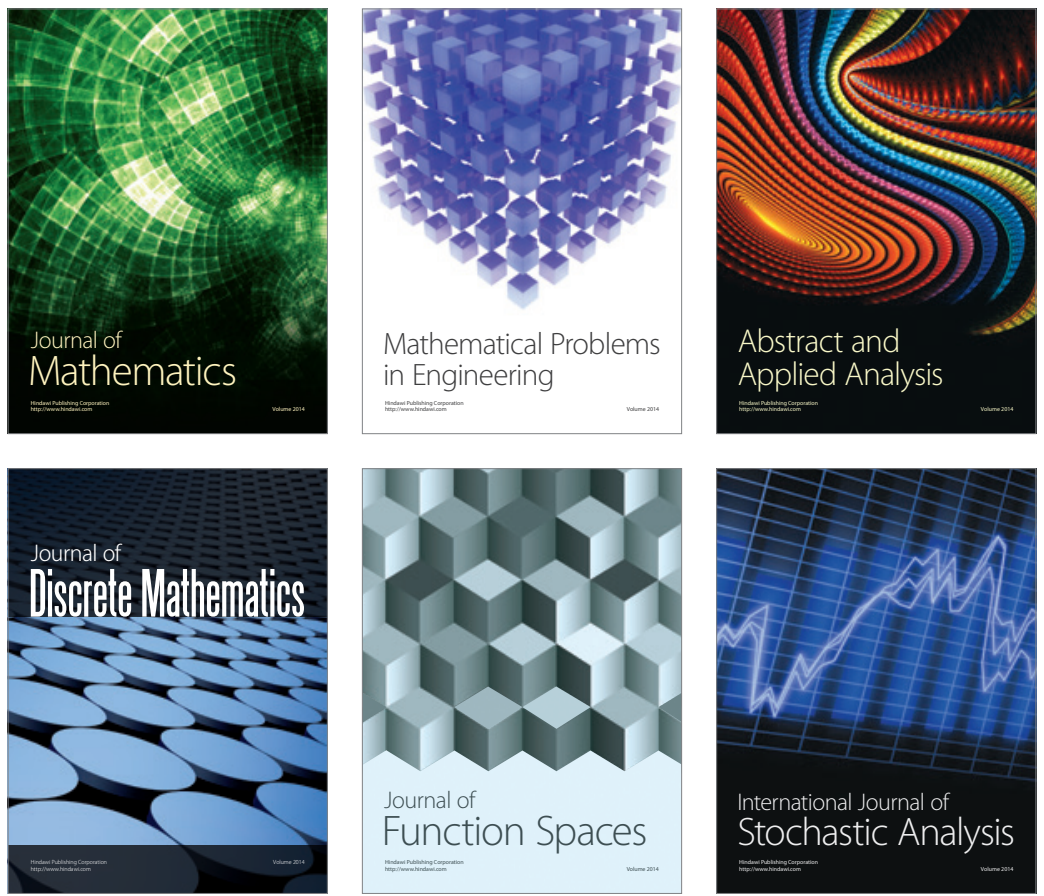

Journal of

Function Spaces

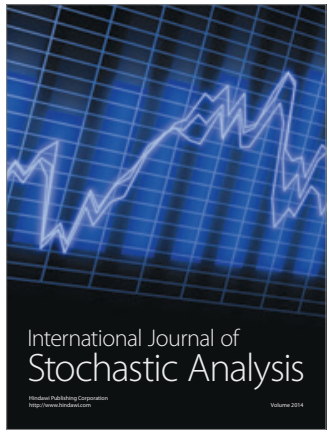

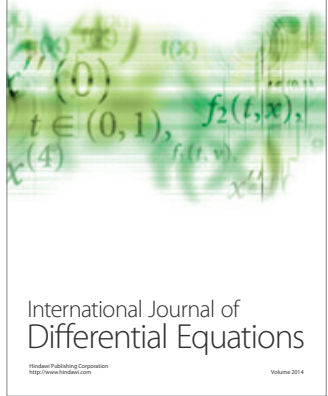
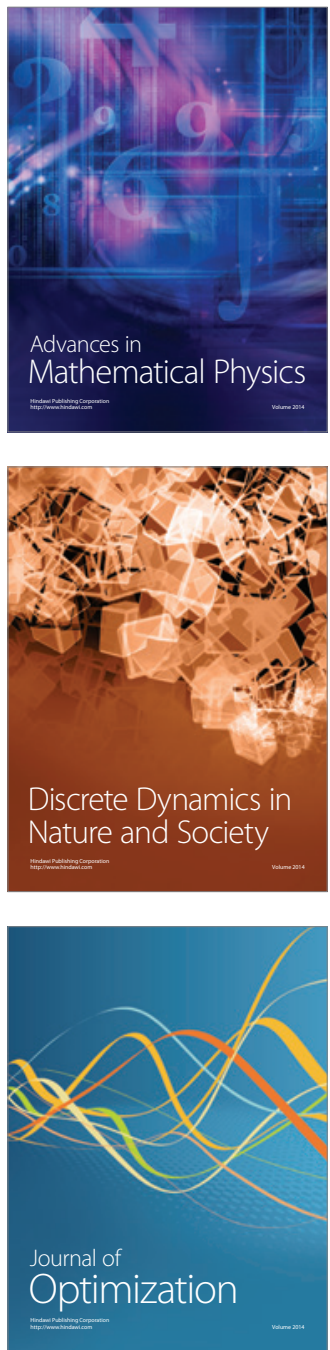